ARTICLE

https://doi.org/10.1038/s41467-019-09233-7

\title{
MERTK mediated novel site Akt phosphorylation alleviates SAV1 suppression
}

\author{
Yao Jiang 1,2,3, Yanqiong Zhang (1) 2,3, Janet Y. Leung ${ }^{2,4}$, Cheng Fan'2,5,6, Konstantin I. Popov ${ }^{3}$, Siyuan Su ${ }^{2,3}$, \\ Jiayi Qian 2,3, Xiaodong Wang 2,7, Alisha Holtzhausen (1) 2,8, Eric Ubil 2,8, Yang Xiang9, Ian Davis 2,5,10, \\ Nikolay V. Dokholyan (10 2,3,11, Gang Wu', Charles M. Perou 2,5,6, William Y. Kim²,4,5,8, H. Shelton Earp ${ }^{2,8}$ \& \\ Pengda Liu 2,3
}

Akt plays indispensable roles in cell proliferation, survival and metabolism. Mechanisms underlying posttranslational modification-mediated Akt activation have been extensively studied yet the Akt interactome is less understood. Here, we report that SAV1, a Hippo signaling component, inhibits Akt, a function independent of its role in Hippo signaling. Binding to a proline-tyrosine motif in the Akt-PH domain, SAV1 suppresses Akt activation by blocking Akt's movement to plasma membrane. We further identify cancer-associated SAV1 mutations with impaired ability to bind Akt, leading to Akt hyperactivation. We also determine that MERTK phosphorylates Akt1-Y26, releasing SAV1 binding and allowing Akt responsiveness to canonical PI-3K pathway activation. This work provides a mechanism underlying MERTK-mediated Akt activation and survival signaling in kidney cancer. Akt activation drives oncogenesis and therapeutic resistance; this mechanism of Akt regulation by MERTK/SAV1 provides yet another complexity in an extensively studied pathway, and may yield prognostic information and therapeutic targets.

\footnotetext{
${ }^{1}$ Cancer Center, Union Hospital, Tongji Medical College, Huazhong University of Science and Technology, Wuhan 430022, China. ${ }^{2}$ Lineberger Comprehensive Cancer Center, The University of North Carolina at Chapel Hill, Chapel Hill, NC 27599, USA. ${ }^{3}$ Department of Biochemistry and Biophysics, The University of North Carolina at Chapel Hill, Chapel Hill, NC 27599, USA. ${ }^{4}$ Department of Medicine, The University of North Carolina at Chapel Hill, Chapel Hill, NC 27599, USA. ${ }^{5}$ Department of Genetics, The University of North Carolina at Chapel Hill, Chapel Hill, NC 27599, USA. ${ }^{6}$ Department of Pathology and Laboratory Medicine, The University of North Carolina at Chapel Hill, Chapel Hill, NC 27599, USA. ${ }^{7}$ Center for Integrative Chemical Biology and Drug Discovery, Division of Chemical Biology and Medicinal Chemistry, Eshelman School of Pharmacy, The University of North Carolina at Chapel Hill, Chapel Hill, NC 27599, USA. ${ }^{8}$ Department of Medicine and Pharmacology, The University of North Carolina at Chapel Hill, Chapel Hill, NC 27599, USA. ${ }_{9}^{9}$ Abclonal Technology, 86 Cummings Park Drive, Woburn, MA 01801, USA. ${ }^{10}$ Department of Pediatrics, The University of North Carolina at Chapel Hill, Chapel Hill, NC 27599, USA. ${ }^{11}$ Present address: Departments of Pharmacology, Biochemistry and Molecular Biology, Pennsylvania State College of Medicine, Hershey, PA 17033, USA. These authors contributed equally: Yao Jiang, Yanqiong Zhang. Correspondence and requests for materials should be addressed to P.L. (email: pengda_liu@med.unc.edu)
} 
A dvances in genomics, gene function annotation, and systems biology have revealed pathways in normal cells that are deranged in cancer; this knowledge serves as a blueprint for targeted cancer therapy ${ }^{1}$. Protein-protein interactions (PPI), often governed by posttranslational modifications, play an essential role in integrating proteins into signal transduction pathways and networks. Although more than 650,000 PPIs have been predicted by large-scale proteomics approaches ${ }^{2-4}$, so far only a few agents such as venetoclax approved to treat patients with chronic lymphocytic leukemia (CLL) directly target PPIs 5 . This is in large part due to the limited association between PPIs and pathophysiological functions.

The oncogenic protein kinase Akt plays essential roles in regulating cell proliferation, survival, metabolism, and genome stability $^{6}$. Hyperactivation of Akt has been observed virtually in all solid tumors ${ }^{7,8}$ and has been shown to drive tumorigenesis in different cancer settings using a variety of murine models ${ }^{9}$. In addition to Akt gene amplification and mutation ${ }^{10}$, various extracellular signals drive posttranslational modifications of Akt in normal and neoplastic cells, controlling Akt activation and oncogenicity, including phosphorylation ${ }^{11-13}$, hydroxylation ${ }^{14}$, acetylation $^{15}$, ubiquitination ${ }^{16,17}$, and others. Accordingly, inhibitors targeting these modifying enzymes have been developed and examined clinically, currently with limited success ${ }^{18}$. In contrast to well-characterized Akt posttranslational modifications identified in the past ${ }^{19}$, our knowledge about how non-enzymatic Akt binding proteins modulate Akt activity in cancer is limited, and whether Akt PPIs can be exploited for cancer therapy remains to be determined. With rapidly developing techniques to make targeting oncogenic PPIs feasible ${ }^{20}$, these findings would shed light into both Akt biology and cancer therapeutics. Here, we identify SAV1 as an Akt endogenous inhibitor and SAV1mediated Akt suppression can be released by either MERTKmediated Akt1-Y26 phosphorylation or by cancerous SAV1 mutations with deficiencies in binding Akt. Thus, our results suggest that both SAV1 and MERTK contribute to Akt activity regulations, and SAV1 is a critical component for MERTK inhibitor-mediated suppression of Akt activation in renal cell carcinoma.

\section{Results}

SAV1 is an endogenous Akt inhibitor: SAV1 binds and suppresses Akt activation. Since the Akt signaling regulates cell size ${ }^{21}$, whereas the Hippo signaling controls cell growth by modulating organ size ${ }^{22}$, we hypothesized that these pathways may be coordinated. Given that WW-domains in various Hippo signaling pathway members, such as SAV1, LATS1, YAP, and TAZ, mediate Hippo signal transduction ${ }^{23}$, we investigated their interactions with Akt finding only one of these key WW-domain containing Hippo components, SAV1, but not others (such as YAP and TAZ), specifically bound Akt1 in cells (Fig. 1a, 1b). Furthermore, this interaction was mediated by the SAV1-WW domain (Supplementary Fig. 1a to 1c) and the Akt1-PH domain (Supplementary Fig. 1d and 1e). Given that the "PxY" motif is a specific WW-domain binding motif ${ }^{24}$, we identified a " $\mathrm{P}_{24} \mathrm{R}_{25} \mathrm{Y}_{26}$ " motif in the Akt1 PH domain (Fig. 1c) that is both evolutionarily conserved and present in all three Akt isoforms necessary for this interaction (Fig. 1d and Supplementary Fig. 1f). A structural simulation using available structures for the Akt1-PH domain and SAV1 suggests that both $\mathrm{P}_{24}$ and $\mathrm{Y}_{26}$ residues reside on the interaction surface between SAV1 and Akt1 (Supplementary Fig. 1g). Consistent with the notion that these residues are critical in mediating Akt1 interaction with SAV1, an Akt1-P24A mutant significantly reduced Akt1 binding to SAV1 (Fig. 1e and Supplementary Fig. $1 \mathrm{~h}$ and 1i). In contrast, an Akt1-Y26F mutation dramatically enhanced SAV1 binding (Fig. 1e and Supplementary Fig. $1 \mathrm{~h}$ and 1i), presumably due to strengthened molecular interaction between SAV1 and Akt1 (Supplementary Fig. 1j and $1 \mathrm{k})$. The fact that Akt1-Y26F is a tyrosine phospho-deficient mutation suggests that phosphorylation of the Akt1-Y26 residue would antagonize SAV1 binding to the Akt1-PH domain. In support of this hypothesis, we observed that Y26-phosphorylated Akt1-PH peptides were deficient in binding SAV1, as compared to non-phosphorylated peptide controls (Fig. 1f). Importantly, the Akt1-Y26F mutation dramatically reduced Akt-pT308 signals in cells (Fig. $1 \mathrm{~g}$ and Supplementary Fig. 1l) and attenuated Akt activity in vitro (Supplementary Fig. $1 \mathrm{~m}$ ), whereas Akt1-P24A exhibited enhanced Akt-pT308 (Supplementary Fig. 11). These data suggest that SAV1 binds directly with the Akt-PH domain to inhibit Akt activity.

Consistent with SAV1 being an Akt activity suppressor, kidney-specific $S A V 1$ deletion in mice ${ }^{25}$ led to elevated AktpT308 (Fig. 1h). Moreover, reduced SAV1 expression was observed in renal cell carcinoma (RCC) patients (Supplementary Fig. 2a). In most pairs of clear cell renal cell carcinoma (ccRCC) patient samples we analyzed, reduced SAV1 expression correlated with high Akt-pT308 signals (Supplementary Fig. 2b). Moreover, consistent with previous reports that chromosomal $14 q$ (the location of SAV1) loss was observed in high-grade ccRCC and neuroblastoma ${ }^{26,27}$, we detected a moderate inverse correlation between SAV1 expression and Akt-pT308 levels in a cohort of RCC patients with stage IV disease (Supplementary Fig. 2c), but not earlier stages (Supplementary Fig. 2d to 2f). Consistently, phosphorylation of an Akt substrate, GSK3 $\beta$ (Supplementary Fig. 2g) also moderately inversely correlated with reduced SAV1 expression in stage IV KIRC dataset, while the association of AktpS473 (Supplementary Fig. 2h) or Akt upstream activating enzymes including mTOR (Supplementary Fig. 2i) and PDK1 (Supplementary Fig. 2j) with SAV1 expression were not noticeably correlated. Notably, no such correlation was observed in KIRP dataset (Supplementary Fig. $2 \mathrm{k}$ to 2o). Interestingly, either low SAV1 expression (Supplementary Fig. $2 p$ and $2 q$ ) or high Akt-pT308 levels (Supplementary Fig. 2r and 2s) predicted worse prognosis and survival in RCC patients. These observations suggest that deficiency in SAV1 may facilitate RCC development in part through promoting Akt activation in RCC patients. This also suggests that SAV1 may be an independent marker distinct from the canonical Akt control mechanisms, given that $S A V 1$ genetic alternations are not coincident with genetic alternations in PIK3CA or PTEN (Supplementary Fig. 2t). These data support that SAV1 may be a bona fide Akt suppressor independent of "classical" Akt regulatory pathways.

Consistent with a previous report ${ }^{28}$, SAV1 expression was not detectable in two ccRCC cell lines, 786-O and ACHN (Fig. 1i). Depletion of endogenous SAV1 in SAV1-expressing RCC cell lines (Fig. 1i), including RCC4, UMRC6, and Caki-1, resulted in dramatically increased Akt-pT308 (Fig. 1j, k and Supplementary Fig. 3a) and enhanced cell growth (Fig. 1l, m). In contrast, ectopic expression of SAV1 in both SAV1-deficient (Supplementary Fig. 3b) and SAV1-proficient RCC (Supplementary Fig. 3c and $3 \mathrm{~d}$ ) and in non-RCC cells (Supplementary Fig. 3e to 3g) led to attenuated Akt-pT308 levels, which was predominately mediated by the SAV1-WW domain (Supplementary Fig. $3 \mathrm{~h}$ and 3i). Notably, no significant changes of PTEN expression were observed upon manipulating SAV1 expression (Fig. 1h, j, 1 and Supplementary Fig. $3 \mathrm{c}$ to $3 \mathrm{f})^{29}$. Our observed SAV1-mediated suppression of Akt activation may not function through MST1, given that multiple upstream signals in addition to MST1/SAV1 have been identified that activate LATS1/2 to suppress YAP/ $\mathrm{TAZ}^{30,31}$, which could compensate for SAV1 deficiency in regulating YAP activity. This is further supported by our 
a

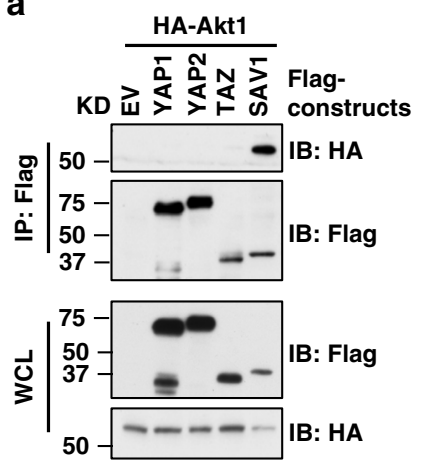

f<smiles>[C+]1[CH-]CCC1</smiles>

pY26: Biotin- GEYIKTWRPRYFLLKNDGTF WT: Biotin- GEYIKTWRPRYFLLKNDGTF

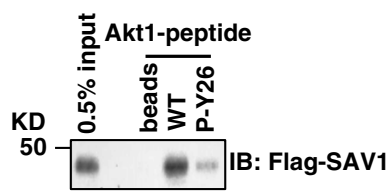

j

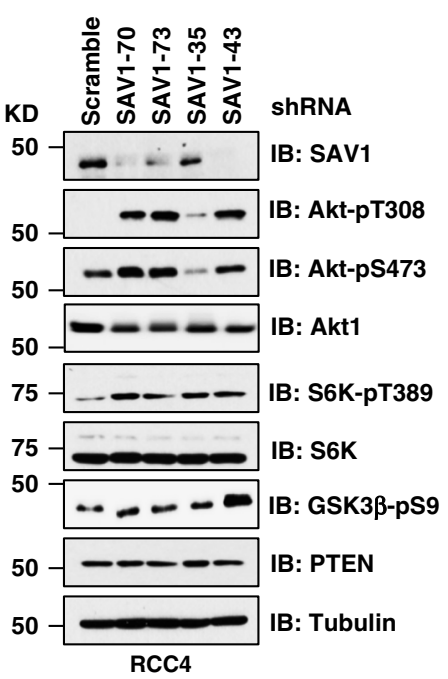

b

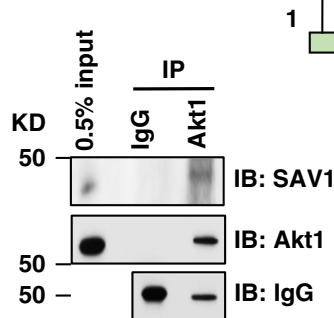

C 23RPRY26

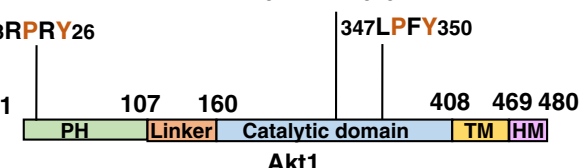

d

homo Akt1 21 TWRPRYFLLKN homo Akt2 21 TWRPRYFLLKS homo Akt3 21 NWRPRYFLLKT mus Akt1 21 TWRPRYFLLKN rat Akt1 21 TWRPRYFLLKN e

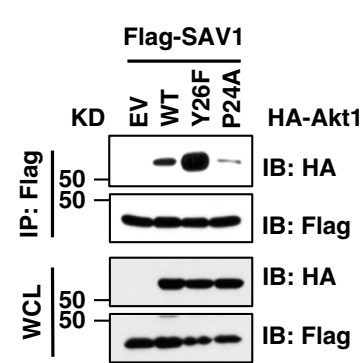

i

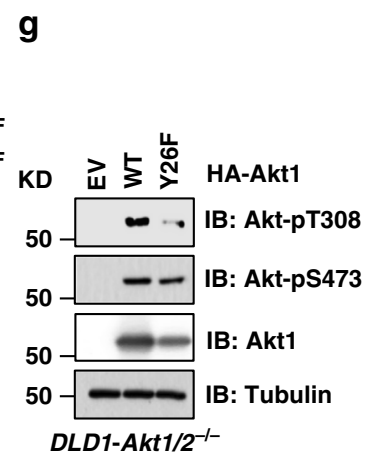

k

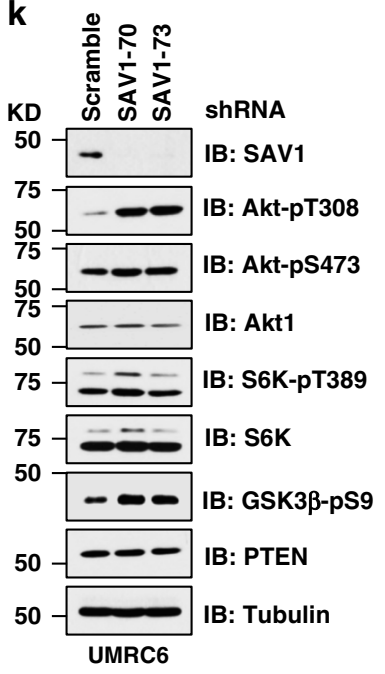

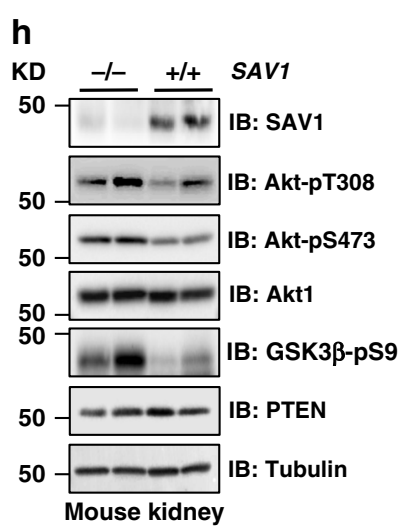

I
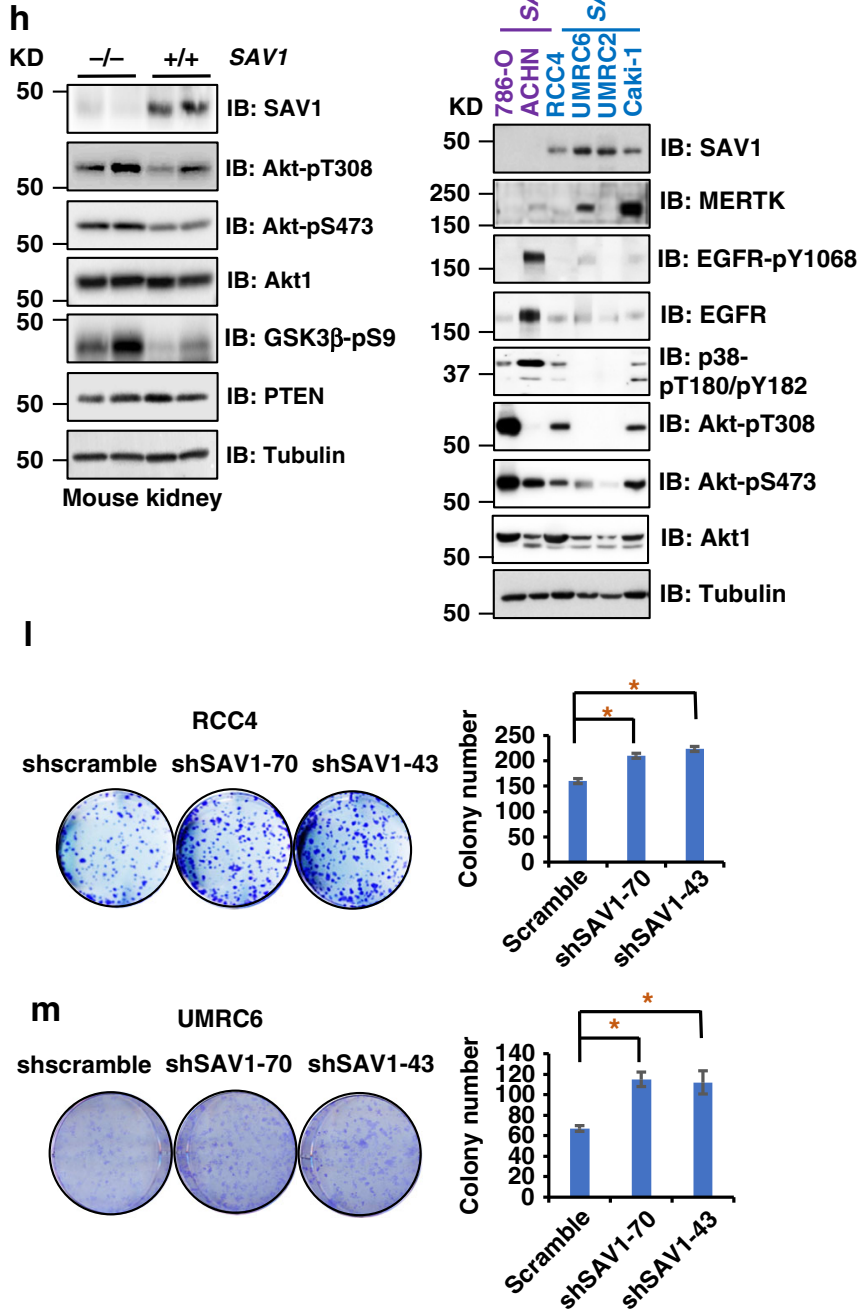

Fig. 1 SAV1 binds and suppresses Akt activity. a Immunoblot (IB) analysis of whole cell lysates (WCL) and Flag-immunoprecipitates (IP) derived from HEK293 cells transfected with indicated DNA constructs. b IB analysis of WCL and endogenous Akt1-IP derived from RCC4 cells. c A cartoon illustration of the distribution of PxY motifs in Akt1. d Protein sequence alignment the "PxY" motif located in the PH domain of Akt isoforms. e IB analysis of WCL and Flag-IP derived from HEK293 cells transfected with indicated DNA constructs. $\mathbf{f I B}$ analysis of indicated Akt1 peptide pulldown products. $\mathbf{g}$ IB analysis of WCL derived from DLD1-Akt1/2-/- cells transfected with indicated HA-Akt1 constructs. $\mathbf{h} I B$ analysis of WCL derived from WT and SAV1-/- mouse kidney tissues. $\mathbf{i}$ IB analysis of WCL derived from indicated RCC lines. $\mathbf{j}, \mathbf{k}$ RCC4 (j) and UMRC6 (k) cells were depleted of endogenous SAV1 by lentiviral shRNAs against SAV1 and harvested 72 -h post puromycin selection $(1 \mu \mathrm{g} / \mathrm{ml}$ ) for IB analysis. $\mathbf{I}, \mathbf{m} 600$ cells resulted from (j) and (k) were subjected to colony formation assays

observation that in $293 \mathrm{~A}$ cells, genetic deletion of neither MST1/2 nor LATS1/2 impaired Akt phosphorylation (Supplementary Fig. 3j). Although both Akt1 and MST1 bind SAV1, they did not appear to compete for SAV1 binding in cells (Supplementary
Fig. 3k and 31), suggesting that SAV1 may not be a rate-limiting factor in cells for Hippo and Akt regulation. Interestingly, an inverse correlation between SAV1 expression and Akt-pT308 was observed in a panel of SAV1-expressing RCC lines 

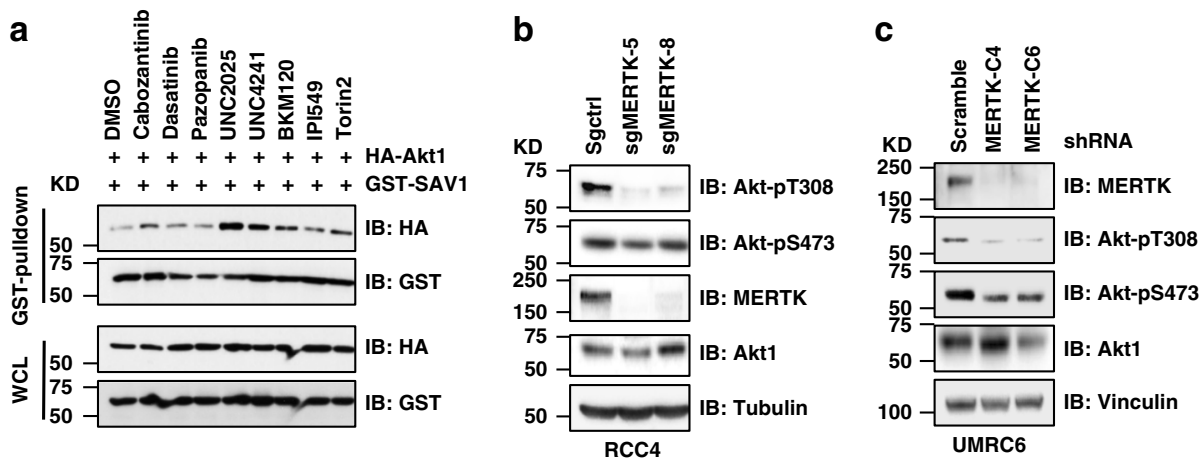

d
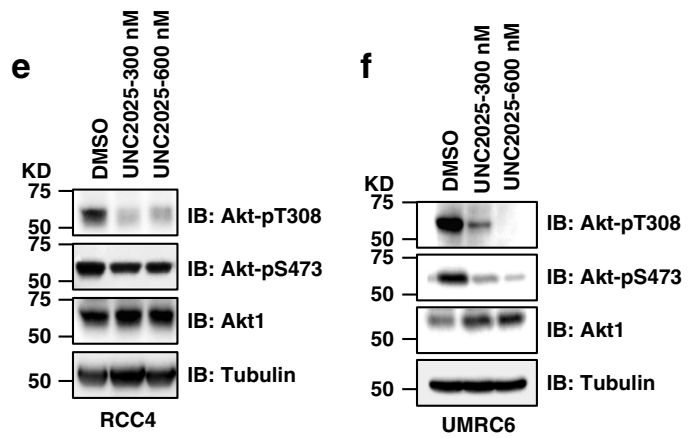

g

h
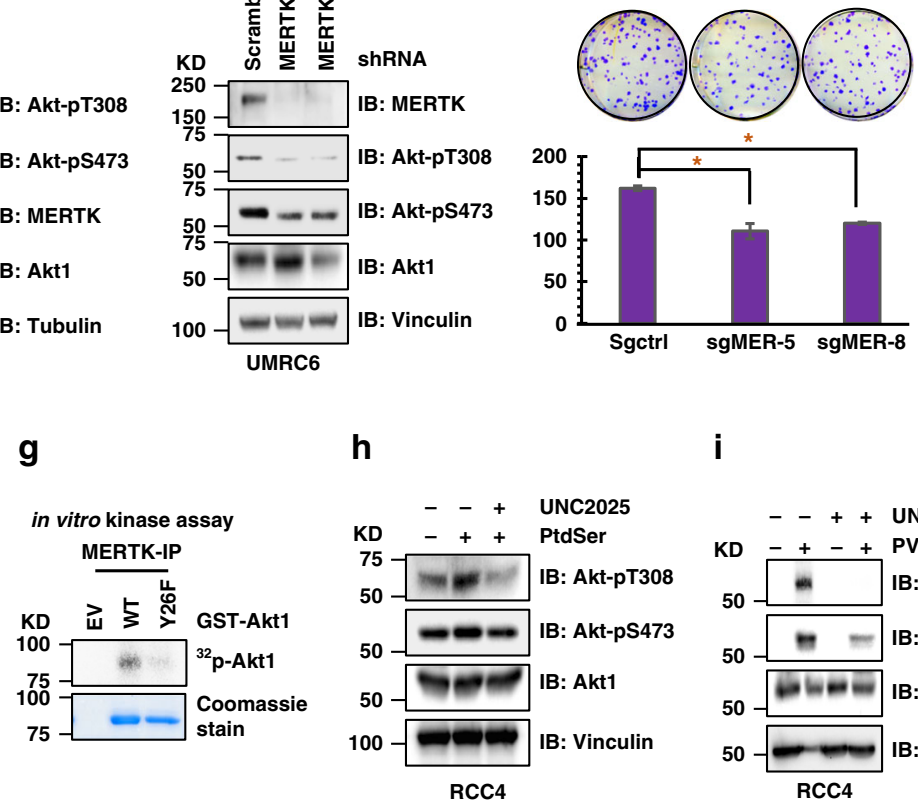

i

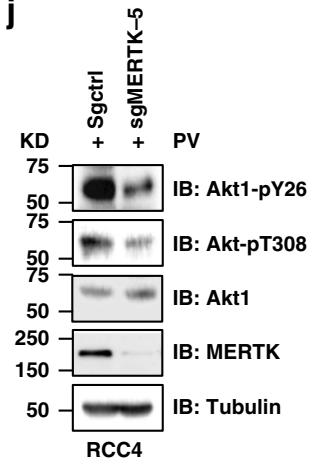

k

I
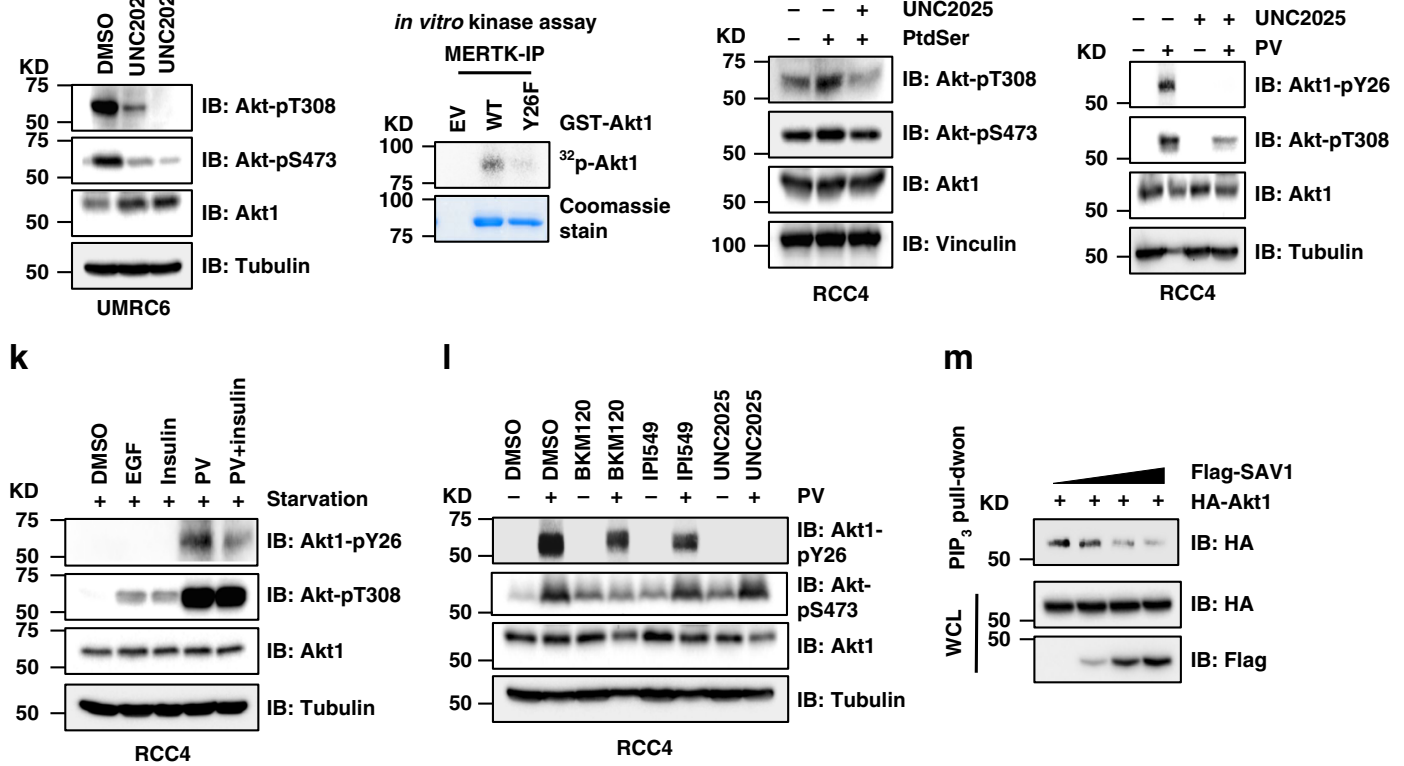

m
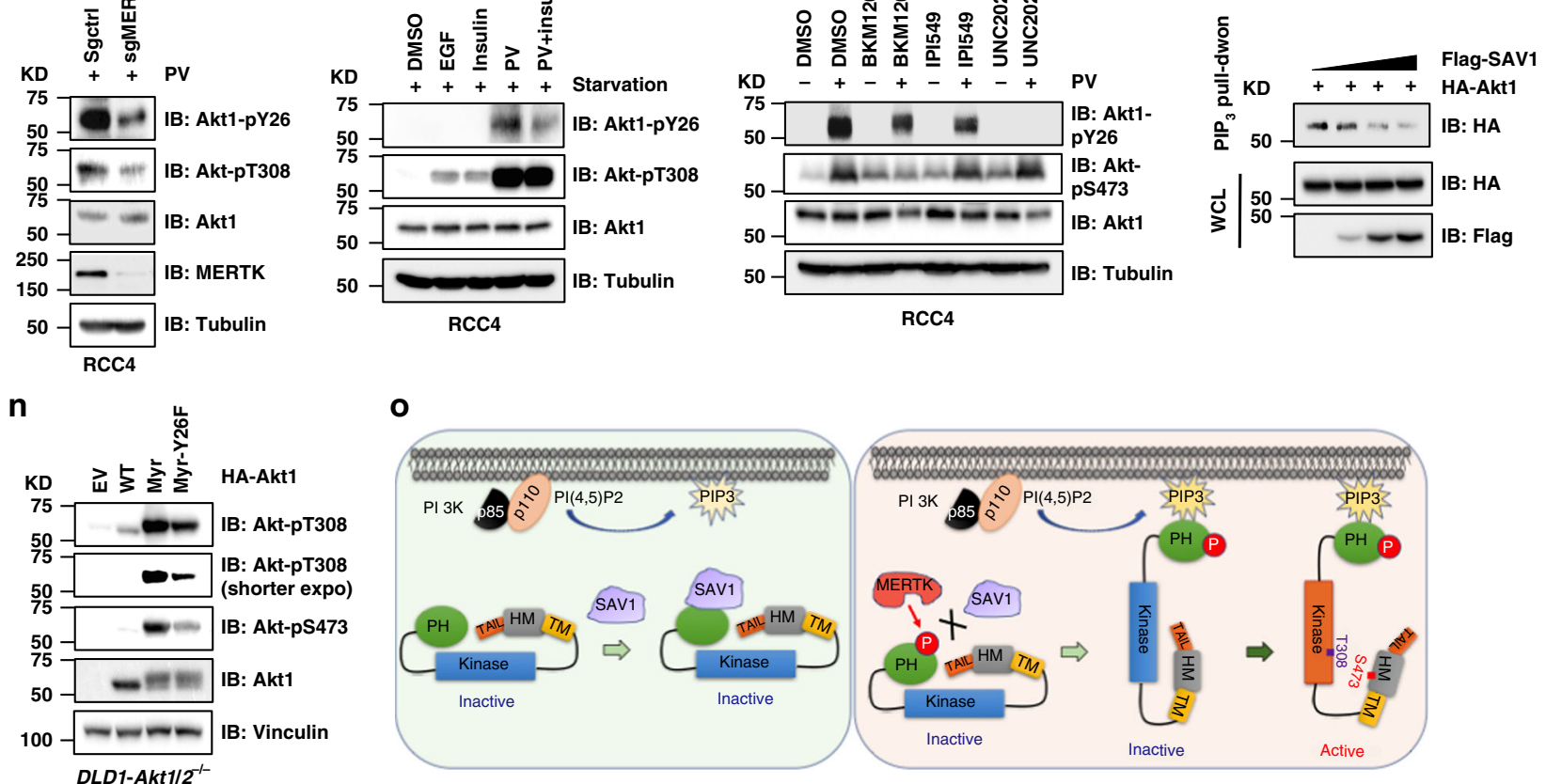

Fig. 2 MERTK phosphorylates and activates Akt by releasing SAV1 binding. a IB analysis of WCL and GST-pulldown products derived from HEK293 cells transfected with CMV-GST-SAV1 and HA-Akt1 constructs and treated with indicated kinase inhibitors for $12 \mathrm{~h}$ before cell collection. Doses for inhibitors used as following: cabozantinib (c-Met and VEGFR2 inhibitor, $100 \mathrm{nM}$ ), dasatinib (Src inhibitor, $100 \mathrm{nM}$ ), pazopanib (VEGFR inhibitor, $100 \mathrm{nM}$ ), UNC2025 (MERTK inhibitor, $300 \mathrm{nM}$ ), UNC4241 (MERTK inhibitor, $300 \mathrm{nM}$ ), BKM120 (PI3K $\alpha$ inhibitor, $100 \mathrm{nM}$ ), IPI549 (PI3K $\gamma$ inhibitor, $100 \mathrm{nM}$ ), and Torin 2 (mTOR inhibitor, $100 \mathrm{nM}$ ). b IB analysis of WCL derived from RCC4 cells deleted of endogenous MERTK using indicated sgRNAs by CRISPR-Cas9. c IB analysis of WCL derived from UMRC 6 cells depleted of endogenous MERTK using indicated shRNAs. $\mathbf{d}$ Deletion of MERTK in RCC4 cells leads to reduced colony formation ability. e, $\mathbf{f} I \mathrm{~B}$ analysis of WCL derived from RCC4 (e) or UMRC6 (f) cells treated with indicated MERTK inhibitors with indicated doses for $2 \mathrm{~h} . \mathbf{g}$ In vitro kinase assays to demonstrate that MERTK directly phosphorylates the Akt1-Y26 in vitro. $\mathbf{h}-\mathbf{j}$ IB analysis of WCL derived from RCC4 cells treated with $80 \mu \mathrm{M}$ phosphatidylserine (PtdSer) (h) or $10 \mu \mathrm{M}$ pervanadate (PV) (i, $\mathbf{j})$ for $30 \mathrm{~min}$ in the absence or presence of $300 \mathrm{nM}$ MERTK inhibitor UNC2025 (h, i) or MERTK deletion by CRISPR (j). $\mathbf{k}$, I IB analysis of WCL derived from RCC4 cells serum starved overnight and then treated with indicated stimulation and inhibitors. EGF (100 ng/ml, $10 \mathrm{~min})$, insulin (100 nM, $30 \mathrm{~min}), \mathrm{PV}(60 \mu \mathrm{M}, 30 \mathrm{~min}), \mathrm{BKM} 120$ (200 nM, $10 \mathrm{~h}), \mathrm{IPI} 549$ (100 nM, $10 \mathrm{~h})$, UNC2025 (300 nM, 2 h). $\mathbf{m}$ IB analysis of WCL and PI(3,4,5) $\mathrm{P}_{3}$ beads pulldown products derived from RCC4 cells transfected with indicated DNA constructs. $\mathbf{n}$ IB analysis of WCL derived from DLD1-Akt1/2-/- cells transfected with indicated HA-Akt1 constructs. $\mathbf{o}$ A cartoon illustration indicating that MERTK-mediated Akt1-Y26 phosphorylation releases SAV1 binding and promotes Akt activation 
(Supplementary Fig. 3m), further supporting the notion that SAV1 is an Akt activity suppressor. Together, these data support that SAV1 binds and suppresses Akt activation independent of its canonical SARAH domain-dependent function in Hippo/YAP regulation (Supplementary Fig. 3n).

Moreover, ectopic expression of SAV1 in 786-O cells $\left(S A V 1^{-1-}\right)$ significantly reduced Akt-T308 phosphorylation in response to EGF (Supplementary Fig. 4a), while depletion of endogenous SAV1 facilitated Akt-T308 phosphorylation upon EGF (Supplementary Fig. $4 \mathrm{~b}$ and $4 \mathrm{c}$ ) or insulin (Supplementary Fig. 4d) stimulation. These data further support that SAV1 suppresses Akt activation under growth factor stimulation conditions.

MERTK phosphorylates Akt to release SAV1 binding. Given that phosphorylation of Akt1-Y26 disrupts SAV1 binding (Fig. 1f), we sought to identify tyrosine kinase(s) that phosphorylates Akt1Y26, which would promote SAV1 release allowing for Akt activation. We reasoned that suppressing the activity of target kinase (s) responsible for Akt1-Y26 phosphorylation would result in enhanced Akt1 interaction with SAV1, mimicking the behavior of the Akt1-Y26F mutation (Fig. 1e). We tested a series of tyrosine kinase inhibitors that target commonly hyper-activated tyrosine kinases in cancer. Interestingly, the broad-spectrum inhibitor dasatinib did not alter the interaction, but MERTK inhibition by two MERTK selective inhibitors (UNC2025 and UNC4241) significantly promoted SAV1 binding to Akt1 (Fig. 2a and Supplementary Fig. 5a and 5b), as well as suppressed Akt-pT308 (Supplementary Fig. 5c) in a Y26 phosphorylation-dependent manner (Supplementary Fig. 5d). Genetic ablation of MERTK by CRISPR (Fig. 2b), or depletion of MERTK by shRNAs (Fig. 2c and Supplementary Fig. 5e), led to significantly reduced Akt-pT308 signals in RCC4 cells and subsequently attenuated RCC4 cell growth (Fig. 2d). The association with MERTK was further supported by reduced Akt-T308 phosphorylation in mertk $^{-1-}$ mouse kidney tissues; the reduction was larger compared with kidney tissues isolated from either tyro3 $3^{-1-}$ or $a x l^{-1-}$ mice (Supplementary Fig. $5 \mathrm{f}$ and $5 \mathrm{~g}$ ). In addition, CRISPR-mediated deletion of Axl or Tyro3, the two other Tyro3/Axl/MerTK (TAM) kinase family members, did not result in significantly reduced Akt-T308 phosphorylation as compared to MERTK (Fig. 2b, c, and Supplementary Fig. $5 \mathrm{~h}$ to $5 \mathrm{k}$ ). Furthermore, deletion of $A x l$ did not retard cell growth (Supplementary Fig. $5 \mathrm{l}$ and $5 \mathrm{~m}$ ). Moreover, acute MERTK inhibition by UNC2025 significantly reduced AktpT308 in both RCC4 (Fig. 2e) and UMRC6 cells (Fig. 2f). In addition, active MERTK could directly phosphorylate Akt1 in vitro mainly on the Y26 residue (Fig. 2g), further supporting MERTK as a major kinase responsible for Akt1-Y26 phosphorylation. Consistent with this notion, a moderate correlation between Akt-pT308 and MERTK activity (evidenced by MEK1pS217/pS221 ${ }^{32}$ ) was observed in Stage IV KIRC patients TCGA dataset (Supplementary Fig. $5 \mathrm{n}$ to $5 \mathrm{q}$ ).

More importantly, phosphatidylserine (PtdSer), the complex physiological activator for MERTK ${ }^{33}$, triggered Akt-pT308 in a MERTK kinase activity-dependent manner (Fig. $2 \mathrm{~h}$ and Supplementary Fig. 6a), in the presence of GAS6 (Supplementary Fig. 6b and $6 \mathrm{c}$ ) produced by RCC4 cells ${ }^{34}$. Moreover, GAS6 also promoted Akt1-pY26 and Akt-pT308 in RCC4 cells (Supplementary Fig. 6d). In keeping with the notion that physiological activation of MERTK promotes Akt activation, we also observed that expression of a constitutively-active MERTK, Fc-MERTK ${ }^{35}$, greatly facilitated Akt-T308 phosphorylation in response to either EGF (Supplementary Fig. 6e) or insulin (Supplementary Fig. 6f) stimulation. On the other hand, MERTK deletion in RCC4 cells resulted in attenuated Akt-T308 phosphorylation in response to either EGF (Supplementary Fig. 6g) or insulin stimulation (Supplementary Fig. 6h). These data cumulatively suggest MERTK as a major kinase phosphorylating Akt1-Y26 for Akt activation. Thus, in the remainder of the study, we mainly focus on understanding the role of MERTK in activating Akt; it remains to be determined whether other RTKs or intracellular TKs also play a role in this process. However, it is interesting that the broad src family TK inhibitor, dasatinib, does not significantly affect SAV1 binding to Akt (Fig. 2a).

To further explore the connection between MERTK phosphorylation of Akt1-Y26 and to explore the physiological function of Akt1-Y26 phosphorylation in cells, we generated an Akt1-pY26specific antibody (Supplementary Fig. 7a and 7b). Pervanadate (PV), a tyrosine phosphatase inhibitor leading to MERTK activation $^{32}$, promoted Akt1-Y26 phosphorylation and AktT308 phosphorylation (Supplementary Fig. 8a to 8c) in a MERTK-dependent manner (Fig. 2i, j and Supplementary Fig. $8 \mathrm{~d}$ and 8e). More importantly, compared with growth factor stimulation by EGF or insulin, PV treatment led to much more significant Akt1-Y26 phosphorylation (Fig. 2k), which could be specifically suppressed by inhibitors targeting MERTK (by UNC2025), but not PI3Ka (by BKM120) nor PI3Ky (by IPI549) (Fig. 21 and Supplementary Fig. 8f). EGFR and insulin receptors are well-known activators of canonical Akt pathways, but confirming their minimal involvement in this mechanism, neither EGF (Supplementary Fig. 9a) nor insulin (Supplementary Fig. 9b) treatment significantly influenced Akt interaction with SAV1. On the other hand, MERTK activation (by Fc-MERTK expression)-induced Akt1-pY26 increase led to attenuated SAV1 binding (Supplementary Fig. 9c).

To further investigate the contribution of the MERTK/Akt/ SAV1 signaling in Akt activation triggered by growth signaling such as PI3K, we ectopically expressed a cancer patient-derived constitutively active H1047R-PIK3CA mutant in RCC cells depleted of endogenous SAV1. Although ectopic expression of either H1047R-PIK3CA or EGFR-L858R promoted basal Akt-T308 phosphorylation (Supplementary Fig. 10a to 10c), in response to growth stimulations including EGF (Supplementary Fig. 10d and 10e) and insulin (Supplementary Fig. 10f), hyperactivation of PI3K did not significantly promote Akt-pT308 signals in SAV1-depleted RCC cells. Similarly, expression of H1047R-PIK3CA also did not functionally compensate for loss of MERTK in promoting phosphorylation of Akt-T308 upon EGF (Supplementary Fig. 10g) or insulin (Supplementary Fig. 10h) stimulation. Moreover, expression of a constitutively active EGFR-L858R mutant similarly did not dramatically promote Akt-pT308 in the absence of neither SAV1 nor MERTK (Supplementary Fig. 10i and 10j) under stimulation conditions in RCC4 cells. Together, these data suggest that MERTK is a major regulator of Akt1-Y26 phosphorylation and subsequent Akt activation, a physiological/potentially pathophysiologic stimulatory pathway distinct from canonical growth factormediated $^{6}$ Akt activation mechanisms.

Next, we examined the molecular mechanism(s) responsible for SAV1 and MERTK-mediated Akt activity control showing that SAV1 binding to the Akt-PH domain (Supplementary Fig. 1e) restrained its accessibility to $\mathrm{PI}(3,4,5) \mathrm{P}_{3}$ (Fig. $2 \mathrm{~m}$ ). However, attenuating Akt plasma membrane recruitment was not the only mechanism through which SAV1 suppresses Akt activation. Introduction of the Y26F mutation into a myristoylation-tagged, active Akt1 that is constitutively attached to the plasma membrane still resulted in reduced Akt activity (Fig. 2n), due to enhanced binding to SAV1 (Supplementary Fig. 11a). Furthermore, we found that enhanced SAV1 binding attenuated Akt binding to its upstream activating kinases, including PDK1 that phosphorylates Akt-T308 ${ }^{12}$ (Supplementary Fig. 11b to 11d) and Sin1 (Supplementary Fig. 11e), an essential mTORC2 component for Akt-S473 phosphorylation $^{11}$ at plasma 


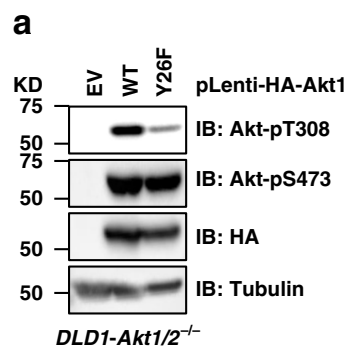

e

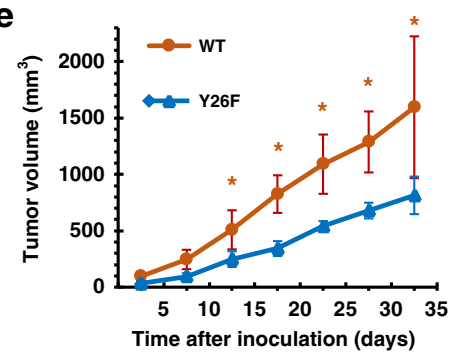

h

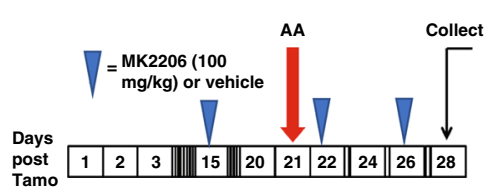

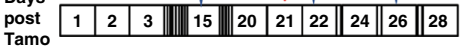

b

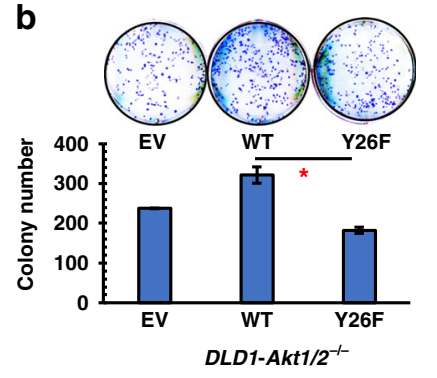

c
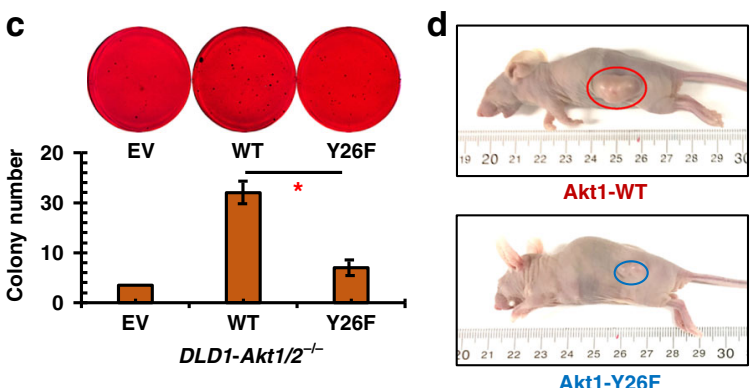

Akt1-Y26F f

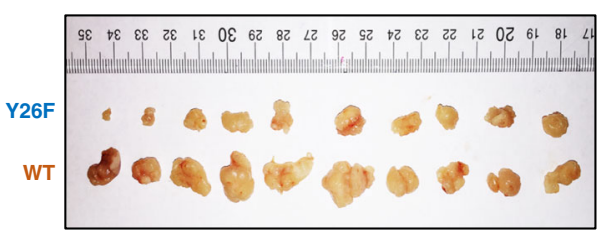

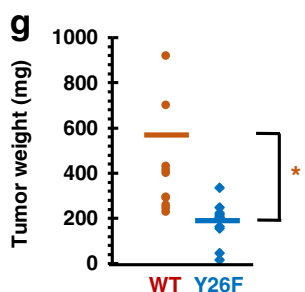

i

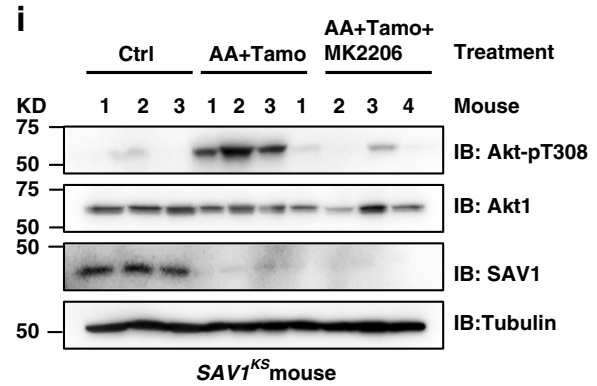

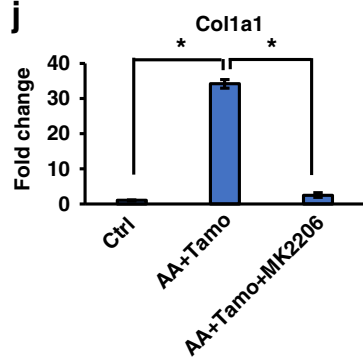

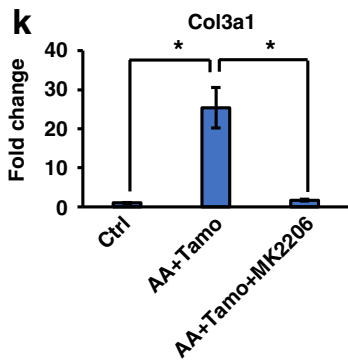

I

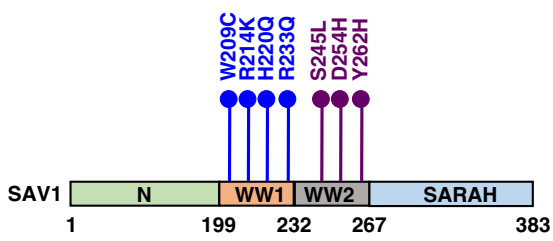

m

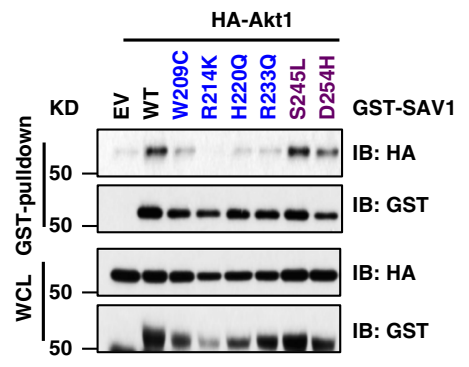

n

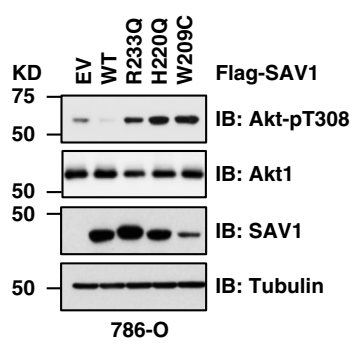

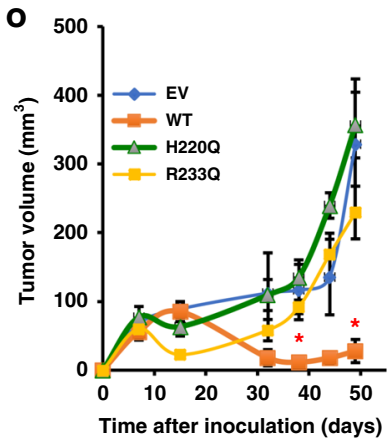

membrane ${ }^{36}$. Thus, SAV1 suppresses Akt activation even once Akt is recruited to the plasma membrane. Together, these data suggest that SAV1 binding not only diminishes Akt recruitment to the plasma membrane lipids but also interferes with subsequent kinase-mediated Akt phosphorylation and activation processes (Fig. 2o). MERTK-mediated Akt-Y26 phosphorylation releases SAV1 binding and suppression, allowing for Akt plasma p

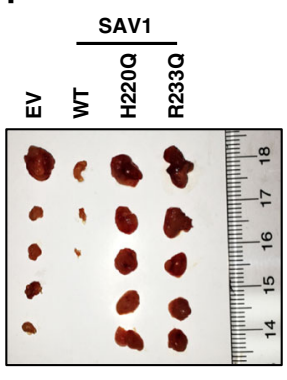

$q$

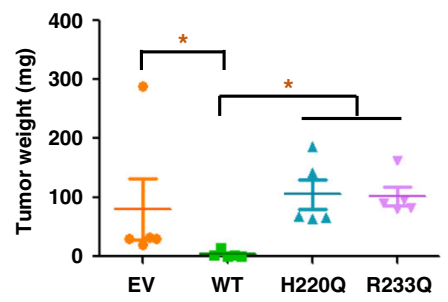

membrane attachment and subsequent phosphorylationmediated activation (Fig. 2o). Together, these data suggest that Akt-pY26 is a control mechanism for Akt activation in SAV1expressing cells.

Akt1-Y26 phosphorylation contributes to Akt oncogenicity. Next, we examined whether enhanced SAV1 binding to Akt, or 
Fig. 3 Akt1-Y26 phosphorylation contributes to the Akt oncogenic capacity. a IB analysis of WCL derived from DLD1-Akt1/2-/- cells infected with lentiviruses encoding indicated Akt1 constructs and selected with $1 \mu \mathrm{g} / \mathrm{ml}$ puromycin for $72 \mathrm{~h}$ before cell collection. b, c Colony formation (b) and soft agar (c) assays were performed with Akt1-WT or Akt1-Y26F expressing DLD1-AKT1/2-/- cells generated in (a), and were quantified (mean \pm SD, $n=3$ ). ${ }^{\star} P<$ 0.05 (Student's $t$ test). d-g Mouse xenograft experiments were performed with the cells generated in (a). Representative tumor images (d), tumor growth curve (e), and tumor weight $(\mathbf{f}, \mathbf{g})$ were calculated (mean $\pm \mathrm{SD}, n=10$ ). ${ }^{\star} P<0.05$ (one-way ANOVA test). $\mathbf{h}$ A schematic representation for drug treatment on $S A V\rceil^{K S}$ mice as indicated. $\mathbf{i} I B$ analysis of $W C L$ derived from $S A V 7^{K S}$ mouse kidneys receiving indicated treatments. $\mathbf{j}, \mathbf{k}$ Analyses of mRNA changes in Col1a1 and Col3a1 fibrosis genes in SAV1KS mouse kidneys receiving indicated treatments. I A schematic representation of cancer patientassociated SAV1 WW-domain mutations. $\mathbf{m}$ IB analysis of WCL and GST-pulldowns derived from HEK293 cells transfected with indicated SAV1-constructs with HA-Akt1. $\mathbf{n}$ IB analysis of WCL derived from 786-O cells (SAV1 ${ }^{-/-}$) transfected with indicated SAV1 constructs. o-q Mouse xenograft experiments were performed with the indicated 786-O cells. Representative tumor growth curve (o), tumor images (p), and tumor weight (q) were calculated (mean \pm $\mathrm{SD}, n=10$ ). ${ }^{\star} P<0.05$ (one-way ANOVA test)

deficiency in MERTK-mediated Akt1-Y26 phosphorylation regulates Akt activity- governed cell growth. We reconstituted DLD1-Akt1/2-l- cells with a stable expression of either WT- or Y26F-Akt1 (Fig. 3a). Consistent with our previous observations, Akt1-Y26F expressing cells displayed significantly reduced AktpT308 signals (Fig. 3a and Supplementary Fig. 12a and 12b), and subsequently attenuated colony formation (Fig. 3b). Moreover, anchorage-independent growth (Fig. 3c) and tumor formation in a mouse xenograft model (Fig. $3 \mathrm{~d}-\mathrm{g}$ ) were also reduced significantly. These data indicate that SAV1 binding to Akt may limit tumorigenesis. Given that we previously found that conditional knockout of Sav1 in mouse renal tubule cells results in renal fibrosis upon acute kidney injury (AKI) with aristolochic acid (AA), concomitant with an oncogene-induced senescence phenotype $^{25}$, next we went on to examine whether it is in part due to Sav1-deletion induced Akt hyperactivation. Consistent with our previous observation, AA treatment in Sav1 ${ }^{K S}$ mice induced expression of genes associated with fibrosis (Supplementary Fig. 12c). More importantly, inhibition of Akt by MK2206 (Fig. 3h, i) significantly suppressed the expression of these fibrosis genes including Collal (Fig. 3j) and Col3al (Fig. 3k). These data strongly support that Akt activation contributes to the genetic and phenotypic manifestations of renal fibrosis induced by AA in Sav1 ${ }^{K S}$ mice and that Akt activation may serve as a major signal downstream of SAV1 deficiency.

Considering that key cell signaling events could be hijacked by cancer to facilitate tumorigenesis ${ }^{37}$, we analyzed TCGA datasets, identified and generated a set of mutations in the WW domains of SAV1 (Fig. 31). Mutations in the functional WW1 domain (W209C, R214K, H220Q, and R233Q), but not in the nonfunctional WW2 domain (S245L and D254H), displayed significantly reduced binding to Akt1 (Fig. 3m) and elevated Akt-pT308 signals (Fig. 3n). Introducing WT-SAV1, but not the H220Q nor R233Q WW1 domain mutant, into 786-O cells (which lack SAV1 expression) suppressed 786-O cell growth both in nude mice (Fig. 3o-q, Supplementary Fig. 12d and 12e) and in vitro (Supplementary Fig. $12 \mathrm{f}$ and $12 \mathrm{~g}$ ). These data strongly suggest that the SAV1-WW1 domain mutations facilitate tumorigenesis potentially through their inability to suppress Akt activation.

\section{Akt suppression by MERTK inhibition requires SAV1 in RCC.} Given that SAV1 binds and suppresses Akt through its $\mathrm{PH}$ domain (Supplementary Fig. 1e), and allosteric Akt inhibitors specifically target the Akt-PH domain to inhibit Akt plasma membrane attachment and activation ${ }^{38}$, we examined whether SAV1 could antagonize the ability of Akt allosteric inhibitors to suppress Akt activity. We found that the allosteric Akt inhibitor, MK2206 (Fig. 4a), but not an ATP analogous inhibitor, GDC0068 (Fig. 4b), specifically competed with SAV1 to bind Akt in an Akt$\mathrm{PH}$ domain-dependent manner (Supplementary Fig. 13a and 13b). Further, SAV1 depletion led to increased cellular sensitivity to MK2206 (Fig. 4c); this was not as evident with GDC0068 (Fig. 4d). These data suggest that reduced SAV1 may sensitize cells to Akt allosteric inhibitors resulting from increased accessibility of Akt-PH domains for these inhibitors.

MERTK plays physiologic roles in innate immune cells and epithelium (including renal epithelia cells ${ }^{39}$ ) sensing PtdSer on apoptotic, ER stressed cells, exosomes, and platelets. It is overexpressed in a variety of neoplastic cells where it provides a survival signal, in part related to Akt activation that has been demonstrated by multiple studies ${ }^{40}$. Examination of the MERTK control of this SAV1 mechanism revealed that when endogenous SAV1 was depleted, MERTK inhibition, which suppressed Akt activation (Fig. 2e, f) now failed to efficiently suppress Akt activation (Fig. 4e and Supplementary Fig. 13c), and these cells were more resistant to MERTK inhibition-mediated growth suppression (Fig. 4f, g), suggesting that SAV1 may be a major downstream effector of MERTK survival signaling in neoplasia and may be a marker to assess whether MERTK inhibition will be effective. Similarly, MERTK inhibition could not further suppress Akt activation when Akt1-Y26 phosphorylation was deficient (Supplementary Fig. 13d), suggesting that MERTK inhibitionmediated Akt suppression is largely through Akt1-Y26 phosphorylation controlled SAV1 loading. Consistent with this notion, unlike WT-SAV1, the cancer-associated SAV1-R233Q mutant was largely resistant to MERTK inhibitor (UNC2025)mediated suppression of Akt activation (Fig. 4h) and displayed less sensitivity to UNC2025 (Fig. 4i).

Together, these data collectively suggest that inhibition of MERTK reduces phosphorylation of Akt1-Y26, which increases SAV1-Akt association (Fig. 4j). This model suggests that cancers lacking SAV1 may have a limited response to MERTK inhibitors, a connection that warrants additional investigations.

\section{Discussion}

Our studies reveal that in spite of intense study of the regulation of a crucial oncogenic driver, Akt, there is still much to learn. Reasoning that cell growth and cell size control might be related we found that SAV1 operates in both pathways with SAV1 being an endogenous Akt inhibitor through its WW domain interaction with a PxY motif in the Akt PH domain. The interaction provides at least two levels of suppression: restriction of $\mathrm{PH}$ binding to the plasma membrane and restriction of interaction with the Akt membrane activating kinases, PDK1 and mTORC2. This additional level of control can be abrogated by tyrosine phosphorylation of the PxY motif, which is much less effectively accomplished by two major kinase systems usually associated with Akt control including EGFR and insulin receptor/IGFR1 ${ }^{6}$. Instead, we demonstrate that MERTK is one RTK that has properties allowing it to regulate this mechanism. Whether other RTKs or TKs can regulate Y26 phosphorylation remains to be determined, but our data suggest that the other two members of the PtdSer sensing RTKs, Axl and Tyro3, are not as efficient in 

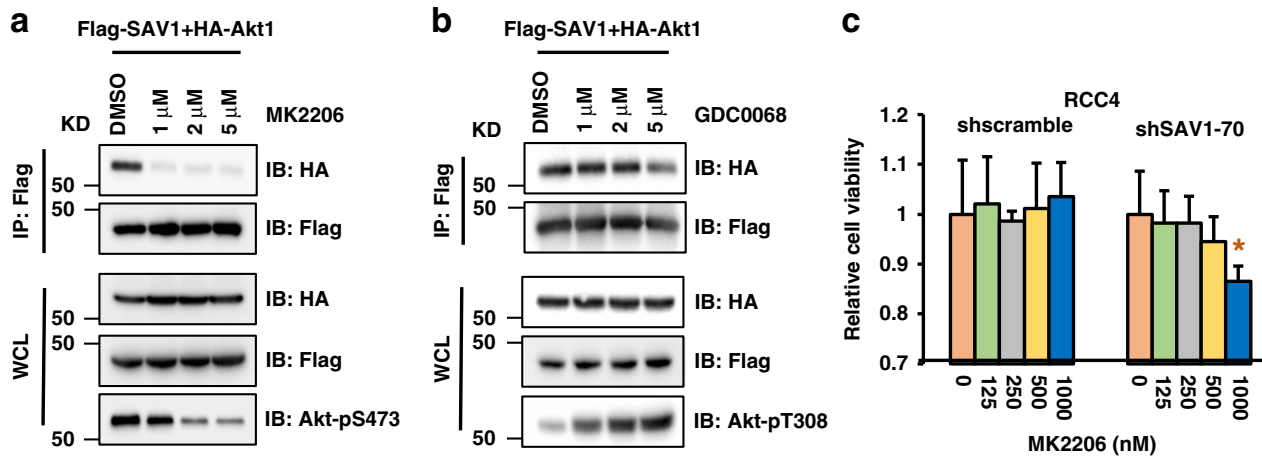

d
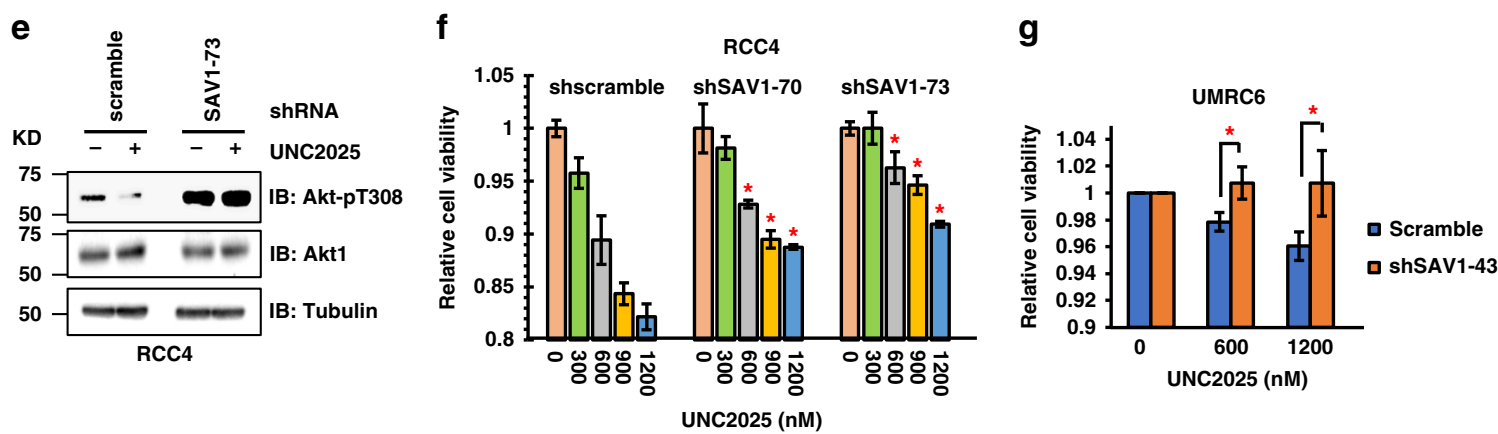

h
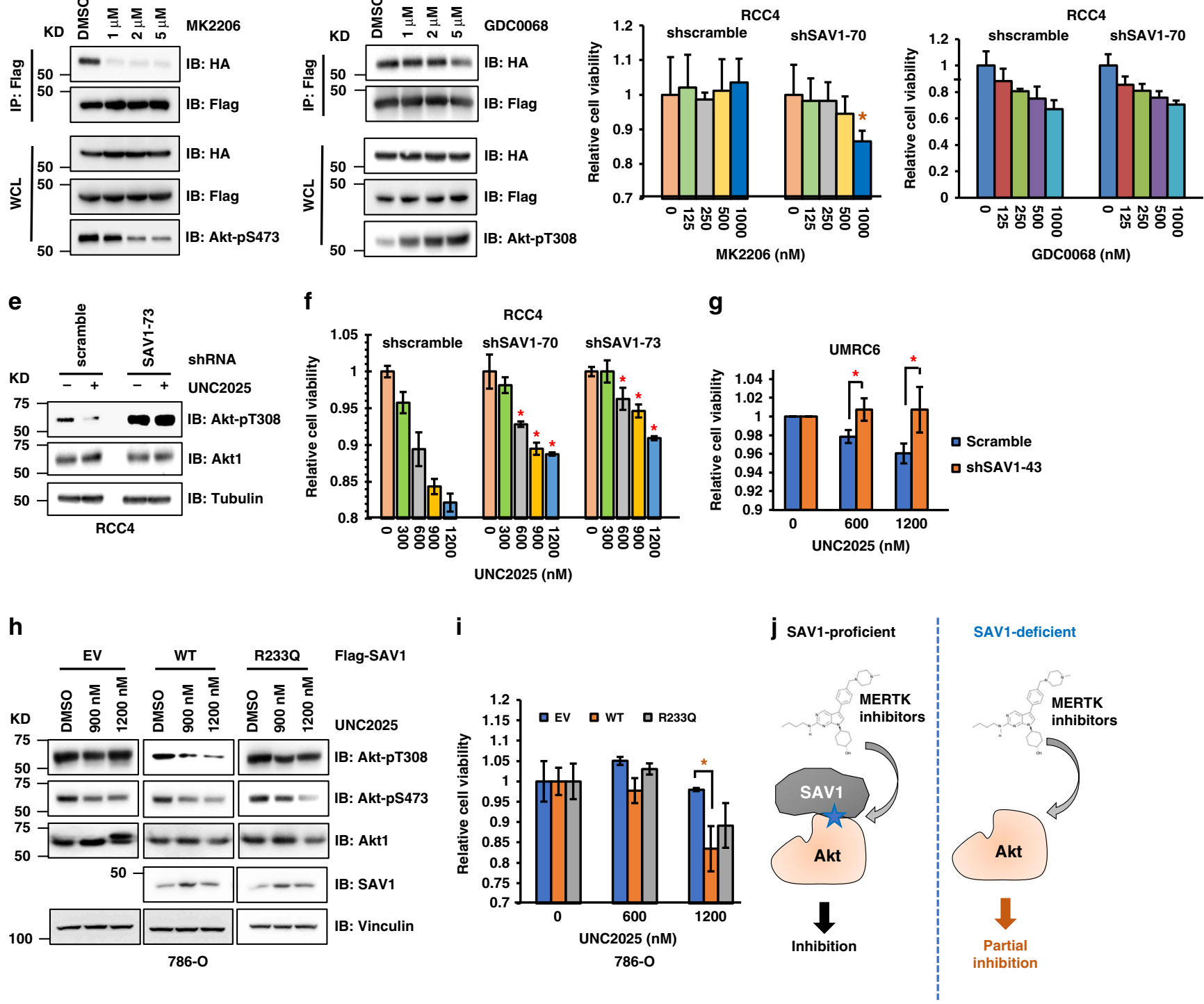

Fig. 4 SAV1 is necessary for MERTK inhibition-mediated suppression of Akt activation in RCC cells. a, $\mathbf{b}$ IB analysis of WCL derived from HEK293 cells transfected with Flag-SAV1 and HA-Akt1. Where indicated, cells were treated with indicated doses of MK2206 (a) or GDC0068 (b) for $10 \mathrm{~h}$ before cell collection. c, d Cell viability assays were performed with WT- and SAV1-depleted RCC4 cells treated with either indicated doses of MK2206 (c) or GDC0068 (d) for 3 days (mean $\pm S D, n=3$ ). 1000 cells were plated in each well on 96 -well plates. ${ }^{\star} P<0.05$ (Student's $t$ test). e IB analysis of $W C L$ derived from indicated RCC4 cells treated with MERTK inhibitor UNC2025 (400 nM) for $2 \mathrm{~h}$ before cell collection. $\mathbf{f}, \mathbf{g}$ Cell viability assays were performed with WT- and SAV1-depleted RCC4 (f) or UMRC6 (g) cells treated with indicated doses of MERTK inhibitor UNC2025 for 3 days (mean \pm SD, $n=3$ ). * $P<$ 0.05 (Student's $t$ test). $\mathbf{h}$ IB analysis of WCL derived from 786-O cells transfected with indicated SAV1 constructs and treated with indicated doses of MERTK inhibitor UNC2025 (400 nM) for $2 \mathrm{~h}$ before cell collection. i Cell viability assays were performed with 786-O cells transfected with indicated SAV1 constructs and treated with indicated doses of MERTK inhibitor UNC2025 for 3 days (mean $\pm S D, n=3$ ). ${ }^{\star} P<0.05$ (Student's $t$ test). j A schematic proposed model to demonstrate that SAV1 is necessary for MERTK-inhibition governed inactivation of Akt. Specifically, when SAV1 expression is high, MERTK inhibitors reduce Akt1-pY26 signals, subsequently enhancing SAV1 binding to Akt to block Akt plasma membrane attachment and binding to Akt upstream activating kinases, thus leading to suppression of Akt. On the other hand, when SAV1 expression is low, MERTK inhibition, although still reduces Akt1-pY26 signals, would achieve limited effects due to the inability to recruit SAV1 for suppressing Akt

carrying out this regulation of a crucial oncogenic driver. Perhaps the larger size of MERTK (170 kDa) compared to Axl ( 138 kDa) and Tyro3 $(\sim 130 \mathrm{kDa})$ gives MERTK a structural/cellular advantage. In epithelial tumors, most of which express MERTK at some level and all of which have abundant apoptotic materials stimulating MERTK activation, this mechanism phosphorylating Akt-Y26, releasing SAV1 (in WT-SAV1 expressing cells), allowing Akt movement to the plasma membrane and unfettered access to PDK1 and mTORC2 would promote oncogenic survival signaling. The biochemical/stoichiometric relationship between the canonical drivers of PI-3K and this mechanism remains to be explored. Notably, cancer therapy-induced cellular apoptosis will lead to accumulation of cellular apoptotic materials including PtdSer, Gas6, and ProteinS, which may contribute to survival advantages essential for therapy resistance. Consistent with this notion, recently activation of the Gas6/TAM signaling upon cytarabine treatment is indispensable for AML resistance to cytarabine $^{41}$. It remains to be further determined whether TAM 
ligands-mediated TAM activation is a general mechanism contributing to cancer therapy-induced therapeutic resistance by promoting Akt-Y26 phosphorylation and Akt activation.

Tumorigenic Akt activation now has other potential mechanisms including deficiency in SAV1 expression, SAV1 mutations in WW domains, which are known to occur ${ }^{26}$, or hyperactivation of MERTK $^{35}$. SAV1 appears to be an important component for MERTK-inhibition triggered Akt inactivation, and the SAV1 expression status could be one marker of whether MERTK inhibitors, which are entering clinical trials, will be efficacious as antitumor cell-directed therapies. Our data also suggest that MERTK inhibitors and allosteric Akt inhibitors will have overlapping sites of action. The Akt pathways are funnels through which almost all cancers pass and as such are major therapeutic targets, the present results implicate MERTK as another potential target to decrease Akt activation. The current study reveals that there are still layers of regulation to be uncovered; starting with a hypothesis that the Akt and Hippo signaling may interact, our studies reveal an additional mechanism and a path by which a less studied RTK, MERTK, may regulate this mechanism. These results could provide a therapeutic target and perhaps a predictive marker to guide clinical trials with MERTK and potentially other TKs that regulate Akt1-Y26 phosphorylation and SAV1/Akt-PH interaction.

\section{Methods}

Cell culture and transfection. HEK293, HEK293T, HeLa, T98G, renal carcinoma cell lines including SAV1-deficient cells (ACHN and 786-O), and SAV1-proficient cells (RCC4 and UMRC6) were cultured in DMEM medium supplemented with $10 \%$ FBS, 100 units of penicillin and $100 \mathrm{mg} / \mathrm{ml}$ streptomycin. HeLa cells were as described in ref. ${ }^{13}$. HEK293T and other RCC cell lines were as described in ref. ${ }^{42}$. DLD1-Akt1 ${ }^{-/-} A k t 2^{-/-}$cells were kindly provided by Dr. Bert Vogelstein (Johns Hopkins University School of Medicine) and these cells also were maintained in DMEM medium supplemented with $10 \%$ FBS. Cell transfection was performed using lipofectamine 2000 or polyethylenimine (PEI) according to manufacture instructions ${ }^{13,36}$. The packaging of lentiviral shRNA or cDNA expressing viruses and retroviral cDNA expressing viruses, as well as subsequent infection of various cell lines were performed according to the adapted protocols ${ }^{14,43}$. Briefly, viral DNA was co-transfected with packaging plasmids including VSVG and $\Delta 8.9$ into HEK293T cells and virus-containing media were collected in 2 consecutive days and used for infection of indicated cells. Following viral infection, cells were maintained in the presence of hygromycin $(200 \mu \mathrm{g} / \mathrm{ml})$, puromycin $(1 \mu \mathrm{g} / \mathrm{ml})$, or blasticidin $(5 \mu \mathrm{g} / \mathrm{ml})$, depending on the viral vector used to infect cells.

Akt kinase inhibitors MK2206 (Selleck S1078) and GDC0068 (Selleck S2808), PI3Ka inhibitor BKM120 (Selleck S2247), mTOR inhibitor Torin 2 (Selleck S2817), PI3K $\gamma$ inhibitor IPI549 (Selleck S8330), c-Met inhibitor cabozantinib (Selleck S1119), Syc inhibitor dasatinib (Selleck S1021), VEGFR inhibitor pazopanib (Selleck S3012), MERTK inhibitors UNC2025 and UNC4241 (produced by UNC drug discovery unit) were used at the indicated dose(s). EGF (Sigma E9644), insulin (Invitrogen 41400-045), pervanadate (Sigma cat\#069K0031), and phosphatidylserine (Avanti, Polar Lipids cat\#840032P) were used at the indicated doses. PIP3 beads (P-B00Ss) were purchased from Echelon Biosciences. Akt1 peptides were synthesized by LifeTin. EGF (1150-04) was purchased from GoldBio. GAS6 was purchased from R\&D Systems (986-GS) or generated by the Earp lab.

Plasmids construction. pcDNA3-HA-Akt1, pcDNA3-HA-Akt2, pcDNA3-HAAkt3, pCMV-Flag-PDK1, and pCMV-Flag-Sin1 were previously described ${ }^{13}$. pCMVFlag-SAV1, pCMV-Flag-TAZ, pCMV-Flag-YAP1, pCMV-Flag-AYP2 were obtained from Dr. Kun-Liang Guan (UC-San Diego). Flag-PIK3CA-WT and Flag-PIK3CAH1047R plasmids were kind gifts from Dr. Alex Toker (Beth Israel Deaconess Medical Center, Harvard Medical School). Fc-MERTK plasmid was obtained from Dr. Raymond Birge (Rutgers University). EGFR-WT and EGFR-L858R plasmids were generated in Dr. H. Shelton Earp lab. pLenti-HA-Akt1-WT and Y26F were constructed by cloning corresponding PCR fragments into pLenti-puro-HA vector described previously ${ }^{13}$ by BamHI and SalI sites. Plenti-HA-SAV1-WT and various mutants were cloned into pLenti6-HA vector by BamHI and XhoI sites. pCMV-GST-SAV1full length and truncation constructs, pCMV-GST-Akt1-full length and truncation constructions were cloned into mammalian expression CMV-GST-fusion vectors. Various Akt1 and SAV1 mutants were generated using the QuikChange XL SiteDirected Mutagenesis Kit (Stratagene) according to the manufacturer's instructions. All mutants were generated using mutagenesis PCR and the sequences were verified by sequencing. Details of plasmid constructions are available upon request.

Akt1-Y26F-Forward: 5'-CATCAAGACCTGGCGGCCACGCTTCTTCCTCCT CAAGAATG-3'

Akt1-Y26F-Reverse: 5'-CATTCTTGAGGAGGAAGAAGCGTGGCCGCCAGG TCTTGATG-3'
Akt1-P24A-Forward: 5'-CATCAAGACCTGGCGGGCACGCTACTTCCTCCT CAAGAATG-3'

Akt1-P24A-Reverse: 5'-CATTCTTGAGGAGGAAGTAGCGTGCCCGCCAGG TCTTGATG-3

Akt1-BamHI-Forward: 5'-GCATGGATCCAGCGACGTGGCTATTGTG-3' Akt1-SalI-Reverse: 5'-GCATGTCGACTCAGGCCGTGCCGCTGGC-3'

shRNAs and sgRNAs. shRNA vectors to deplete endogenous SAV1 were purchased from Sigma (SHCLNG-NM_021818). shRNA vectors to deplete endogenous MERTK were purchased from Sigma (SHCLNG-NM_006343). The sequence for sgRNAs for CRISPR-Cas9 mediated deletion of MERTK were generated by cloning the annealed sgRNAs into BsmBI-digested pLenti-CRISPRv2 vector (Addgene 52961). The short guide RNAs (sgRNA) were designed based on predictions from crispr.mit.edu and oligonucleotides were obtained from Eton Biosciences. Lentiviruses were produced by PEI transfection of $8 \times 10^{6} \mathrm{HEK} 293 \mathrm{~T}$ cells per $10 \mathrm{~cm}$ plate with $2.5 \mu \mathrm{g}$ of target plasmid combined with $1.25 \mu \mathrm{g}$ of VSVG and $1.25 \mu \mathrm{g}$ of $\Delta 8.9$ for transfection. CRISPR sgRNAs were designed as listed below:

sgControl-Forward: 5' -CACCGCTTGTTGCGTATACGAGACT-3';

sgControl-Reverse: 5' -AAACAGTCTCGTATACGCAACAAG-3';

MERTK-sg1-Forward: 5'-CACCGGTAATTTCTCTCCGGACGGA-3';

MERTK-sg1-Reverse: 5'-AAACTCCGTCCGGAGAGAAATTACC-3';

MERTK-sg2-Forward: 5'-CACCGCCCGGGAATAGCGGGTAAGG-3';

MERTK-sg2-Reverse: 5'-AAACCCTTACCCGCTATTCCCGGGC-3';

Axl-sg1-Forward: 5'-CACCG CTGCGAAGCCCATAACGCCA-3';

Axl-Sg1-Reverse: 5'-AAAC TGGCGTTATGGGCTTCGCAG C-3';

Axl-sg2-Forward: 5'-CACCG CGGGCACCTGTGATATTCCC-3';

Axl-Sg2-Reverse: 5'-AAAC GGGAATATCACAGGTGCCCG C-3';

Axl-sg3-Forward: 5'-CACCG GAGAGCCCCCCGAGGTACAT-3';

Axl-Sg3-Reverse: 5'-AAAC ATGTACCTCGGGGGGCTCTC C-3';

Axl-sg4-Forward: 5'-CACCG CAGAGCCCGTGGACCTACTC-3';

Axl-Sg4-Reverse: 5'-AAAC GAGTAGGTCCACGGGCTCTG C-3';

Tyro3-sg1-Forward: 5'-CACCG GCTCTGACGCCGGCCGGTAC-3';

Tyro3-sg1-Reverse: 5'-AAAC GTACCGGCCGGCGTCAGAGC C-3';

Tyro3-sg3-Forward: 5'-CACCG CCCTTTCCAACTGTCTTGTG-3';

Tyro3-sg3-Reverse: 5'-AAAC CACAAGACAGTTGGAAAGGG C-3';

Tyro3-sg5-Forward: 5'-CACCG TGTGAAGCTCACAACCTAAA-3';

Tyro3-sg5-Reverse: 5'-AAAC TTTAGGTTGTGAGCTTCACA C-3'

Antibodies. All antibodies were used at a 1:1000 dilution in TBST buffer with 5\% non-fat milk for western blotting if not otherwise indicated. Anti-SAV1 antibody (cat\#13301, lot\#1), anti-phospho-Thr308-Akt antibody (cat\#2965, lot\#18), antiphospho-Ser473-Akt antibody (cat\#4060, lot\#19 and 23), anti-Akt1 antibody (cat\#2938, lot\#4; and cat\#2967, lot\#17), anti-phospho-Ser9-GSK3b antibody (cat\#5558, lot\#6), anti-PTEN antibody (cat\#9559, lot\#17), anti-phospho-Thr389S6K antibody (cat\#9234, lot\#11), anti-S6K1 antibody (cat\#2708), anti-GST antibody (cat\#2625, lot\#7), anti-phospho42/44-ERK (cat\#4370, lot\#17), anti-phosphoThr180/Tyr182-p38 (cat\#9211, lot\#21), anti-Axl antibody (cat\#8661s, lot\#4), antiPhospho-Y702-Axl (cat\#5724s, lot\#1), anti-Tyro3 antibody (cat\#5585, lot\#2), and polyclonal anti-phospho-Tyr1086-EGFR antibody (cat\#2220s, lot\#2) were purchased from Cell Signaling Technology. Polyclonal anti-HA antibody (cat\#sc-805 lot\#K1215) was obtained from Santa Cruz. Polyclonal anti-Flag antibody (cat\#F7425, lot\#078M4886V), monoclonal anti-Flag antibody (cat\#F-3165, clone M2, lot\#SLBN8915V), monoclonal anti-Tubulin antibody (cat\#T-5168,

lot\#115M4828V), monoclonal anti-Vinculin antibody (cat\#V9131), anti-Flag agarose beads (cat\#A-2220, lot\#SLBW1929), anti-HA agarose beads (cat\#A-2095, lot\#057M4864V), peroxidase-conjugated anti-mouse secondary antibody (cat\#A4416, lot\#SLBW4917), and peroxidase-conjugated anti-rabbit secondary antibody (cat\#A-4914, lot\#SLBV6850) were obtained from Sigma. Anti-MERTK and antiEGFR antibodies were generated by the Earp lab.

The polyclonal Akt1-pY26 antibody was generated by Abclonal, Inc. and was derived from rabbits. We have validated this antibody by dot blotting assays with synthetic non-phosphorylated and Y26-phosphorylated Akt1 peptides, as well as in immunoprecipitations and whole cell lysates from Akt1-WT and Akt1-Y26F constructs transfected into HEK293 cells.

Immunoblot and immunoprecipitations analyses. Cells were lysed in EBC buffer (50 mM Tris, $\mathrm{pH} 7.5,120 \mathrm{mM} \mathrm{NaCl}, 0.5 \% \mathrm{NP}-40)$ or Triton X-100 buffer (50 mM Tris, pH 7.5, $150 \mathrm{mM} \mathrm{NaCl}, 1 \%$ Triton X-100) supplemented with protease inhibitors (Complete Mini, Roche) and phosphatase inhibitors (phosphatase inhibitor cocktail set I and II, Calbiochem). The protein concentrations of whole cell lysates were measured by NanoDrop OneC using the Bio-Rad protein assay reagent according to manufacture instructions ${ }^{13}$. Equal amounts of whole cell lysates were resolved by SDS-PAGE and immunoblotted with indicated antibodies. For immunoprecipitations analysis, $1000 \mu \mathrm{g}$ lysates were incubated with the indicated antibody $(1-2 \mu \mathrm{g})$ for $3-4 \mathrm{~h}$ at $4{ }^{\circ} \mathrm{C}$ followed by $1 \mathrm{~h}$ incubation with $10 \mu \mathrm{l}$ Protein $\mathrm{A}$ magnetic beads (New England Biolabs). Or $1000 \mu \mathrm{g}$ lysates containing tagged molecules were incubated with agarose beads coupled antibodies for the specific tag for $3-4 \mathrm{~h}$ at $4{ }^{\circ} \mathrm{C}$. The recovered immuno-complexes were washed five times with NETN buffer (20 mM Tris, pH 8.0, $100 \mathrm{mM} \mathrm{NaCl}, 1 \mathrm{mM}$ EDTA and $0.5 \% \mathrm{NP}-40$ ) 
before being resolved by SDS-PAGE and immunoblotted with indicated antibodies. Uncropped images are provided in Supplementary Fig. 14.

In vitro Akt kinase assays. Akt in vitro kinase assays were adapted from a protocol described previously ${ }^{13}$. Briefly, $2 \mu \mathrm{g}$ of immune-precipitated HA-Akt1WT or HA-Akt1-Y26F from HEK293 cell lysates were incubated in the presence of $1 \mu \mathrm{Ci}^{3}{ }^{32} \mathrm{P}-\mathrm{ATP}$ and $200 \mu \mathrm{M}$ cold ATP in the kinase reaction buffer (New England Biolabs B6022) for $30 \mathrm{~min}$ at $30^{\circ} \mathrm{C}$. The reaction was stopped by the addition of SDS-containing buffer and resolved by SDS-PAGE. Akt auto-phosphorylation was detected by autoradiography.

In vitro MERTK kinase assays. MERTK in vitro kinase assays were adapted from a protocol ${ }^{13}$. Briefly, $2 \mu \mathrm{g}$ of purified GST-Akt1-WT-K179M or GST-Akt1-Y26FK179M from HEK293 cells were incubated with immunoprecipitated MERTK by MERTK antibodies from RCC4 cells in the presence of $1 \mu \mathrm{Ci}-{ }^{32} \mathrm{P}$-ATP and $200 \mu \mathrm{M}$ cold ATP in the kinase reaction buffer (New England Biolabs B6022) for 30 min at $30{ }^{\circ} \mathrm{C}$. The reaction was stopped by the addition of SDS-containing buffer and resolved by SDS-PAGE. MERTK-mediated Akt phosphorylation was detected by autoradiography.

Peptide synthesis. All peptides were synthesized at LifeTin. Each contained an Nterminal biotin and free C-terminus and was synthesized in the $1-4 \mathrm{mg}$ scale. The sequences are listed below:

Biotin-Akt1-WT: Biotin-GEYIKTWRPRYFLLKNDGTF

Biotin-Akt1-Y26p: Biotin-GEYIKTWRPR-pY-FLLKNDGTF

Dot immunoblot assays. Peptides were spotted onto nitrocellulose membrane allowing the solution to penetrate (usually $3-4 \mathrm{~mm}$ diameter) by applying it slowly as a volume of $2 \mu \mathrm{l}$. The membrane was dried, and blocked in non-specific sites by soaking in TBST buffer with 5\% non-fat milk for immunoblot analysis ${ }^{13}$.

Peptide-binding assays. Indicated biotin-labeled peptides $(2 \mu \mathrm{g})$ were incubated with $1 \mathrm{mg}$ of whole cell lysates prepared from cells in a total volume of $500 \mu \mathrm{l}$ for 4 $\mathrm{h}$ at $4^{\circ} \mathrm{C}$ and $10 \mu \mathrm{l}$ Streptavidin agarose beads (Thermo Scientific 20353) were added for incubation for another $1 \mathrm{~h}$. The agarose was washed four times with NETN buffer. Bound proteins were eluted by boiling in SDS loading buffer, and resolved by SDS-PAGE for subsequence western blotting.

Computational modeling. We used various computational techniques to identify a structural model for Akt1/Savl complex. We started our modeling by performing protein-protein docking of the proteins using ClusPro server ${ }^{44-48}$. Protein Data Bank entrees $1 \mathrm{H} 10^{49}$ and 2YSB (to be published) were used as initial structures of Aktl and Sav1, respectfully. Based on the proximity to expected binding region three docking models were selected. For further optimization and equilibration of the models, we performed a series of low temperature discrete molecular dynamics (DMD) simulations of the protein complexes. DMD is a computationally efficient approach for molecular dynamics simulation of biological molecules ${ }^{50,51}$. It has enhanced sampling capability compare to traditional molecular dynamics approaches due to efficient even based integration algorithms $\mathrm{s}^{52}$. For each docking model equilibrium representative was chosen by performing distance base clustering of all structures obtained during 106 time steps of DMD simulations. The centroids of most populated lowest energies clusters are selected as representatives for our equilibrium models.

In order to select one of the models we used our in-house developed tool Eris to compute changes in stability of the complexes due to mutations: Y26F, W209C, $\mathrm{R} 214 \mathrm{~K}, \mathrm{H} 220 \mathrm{Q}$ and compare them to experimental data. Eris $^{53}$ is a computationally efficient algorithm that uses a physically based force field with atomic resolution and computationally optimized side chains repacking and backbone relaxation algorithms ${ }^{54}$. This approach allows for an accurate calculation of the effect of one or several mutations on protein stability, $\Delta \Delta G$. The model that mostly satisfied with the experimental data is presented in Supplementary Fig. 1g. To study the effect of the Akt1-Y26F mutation we analyzed the dynamics of the Akt1 Sav1 complex on 50 ns timescale by performing DMD simulations for both WT and Y26F mutant. We found that the Y26F mutation increases the hydrophobic surface on the interface of the Akt1/SAV1 complex that promotes rearrangement of the binding interface and results in increasing the number of polar interactions stabilizing the Akt1/Y26F-SAV1 complex (Supplementary Fig. $1 j$ and $1 \mathrm{k})$.

TCGA data analysis. Scatter plot and Pearson correlation analyses were performed for SAV1 mRNA expression vs. Akt-pT308, Akt-pS473, GSK3-pS9, mTOR, PDK1, and other RPPA using TCGA KIRC data, in Stage I, II, III, IV patient samples, respectively. Similar analyses were performed in TCGA KIRP data (without stage information). In addition, Spearman correlation was also performed for SAV1 mRNA expression vs. Akt-pT308 RPPA, Akt-pS473 RPPA, and others as indicated in Stage IV KIRC, where the data from SAV1 mRNA expression vs. AktpT308 RPPA and SAV1 mRNA expression vs. GSK3-pS9 RPPA showed moderate correlations. Moreover, survival analysis was performed for SAV1 mRNA expression and Akt-pT308 RPPA using TCGA KIRC data. The survival analysis is significant in all the data, but not significant in Stage I, II, III, IV separately.

RT-PCR. RT-PCR primers for detection of GAS6 mRNAs are as below:

hGAS6-RT-PCR-F1: 5'-GCAAAACCTGCCTGACCAGT-3'

hGAS6-RT-PCR-R1: 5' -TTCGTTGACATCTTTGTCGCA-3'

$\beta$-actin-F: $5^{\prime}$-CCTGGCACCCAGCACAAT-3'

$\beta$-actin-R: 5'-GCCGATCCACACGGAGTA-3'

RT-PCR experiments were performed as below:

Total cellular RNA was extracted using RNeasy mini kit (QIAGEN) according to the manufacturer's protocol and quantified by a spectrophotometer (Nanodrop One C). cDNA was synthesized using iScript ${ }^{\mathrm{TM}}$ Reverse Transcription Supermix for RT-qPCR (Bio-Rad Cat\#1708841). cDNA templates and iTaq ${ }^{\text {TM }}$ universal SYBR Green Supermix (Bio-Rad Cat\#1725122) were mixed together and the RTPCR reaction was performed on the ViiA ${ }^{\mathrm{TM}} 7$ Real-Time PCR system. The expression of GAS6 and PROS mRNAs was normalized to the expression of $\beta$ actin.

Colony formation assays. Indicated cells were seeded into 6-well plates (300 or 600 cells/well) and cultured in $37^{\circ} \mathrm{C}$ incubator with $5 \% \mathrm{CO}_{2}$ for $\sim 14$ days until the formation of visible colonies. Colonies were washed with $1 \times \mathrm{PBS}$ and fixed with $10 \%$ acetic acid $/ 10 \%$ methanol for $30 \mathrm{~min}$, stained with $0.4 \%$ crystal violet in $20 \%$ ethanol for $30 \mathrm{~min}$, and washed by tap water and air-dried. Colony numbers were manually counted. At least two independent experiments were performed to generate the error bars.

Soft agar assays. The anchorage-independent cell growth assays were performed according to adapted protocols ${ }^{13,36}$. Briefly, the assays were preformed using 6 -well plates where the solid medium consists of two layers. The bottom layer contains $0.8 \%$ noble agar and the top layer contains $0.4 \%$ agar suspended with $1 \times 10^{5}$ or $3 \times$ $10^{4}$ cells. $500 \mu \mathrm{l}$ complete DMEM medium with $10 \%$ FBS was added every 4 days About 4 weeks later the cells were stained with iodonitrotetrazolium chloride (1 $\mathrm{mg} / \mathrm{ml}$ ) (Sigma I10406) overnight for colony visualization and counting. At least two independent experiments were performed to generate the error bar.

Mouse xenograft assays. Mouse xenograft assays were performed according to adapted protocols $^{13,36}$. Briefly, for mouse xenograft experiments in Fig. $3 \mathrm{~d}-\mathrm{g}, 2 \times 10^{6}$ DLD $1-A k t 1 / 2^{-I-}$ cells stably expressing WT or Y26F mutant form of Aktl were injected into the flank of 10 female nude mice (NCRNU-M-M from UNC Animal Facility, 4 weeks old). Tumor size was measured every 3 days with a digital caliper, and the tumor volume was determined with the formula: $L \times W^{2} \times 0.52$, where $L$ is the longest diameter and $W$ is the shortest diameter. After 18 days, mice were sacrificed, and tumors were dissected and weighed. For mouse xenograft experiments in Fig. 3o-q $2 \times 10^{6} 786-\mathrm{O}$ cells expressing indicated SAV1 mutant forms were injected into the flank of indicated male nude mice (NCRNU-M-M from UNC Animal Facility, 4 weeks old). Tumor size was measured with a digital caliper at indicated days post-injection, and the tumor volume was determined with the formula: $L \times W^{2} \times 0.52$, where $L$ is the longest diameter and $W$ is the shortest diameter. After 49 days, mice were sacrificed, and tumors were dissected and weighed.

RNA extraction and cDNA synthesis. Cortex tissue was homogenized in RLT buffer (RNeasy kit; Qiagen) by use of a TissueRuptor homogenizer (Qiagen). RNA was extracted using a RNeasy kit (Qiagen) following the manufacturer's protocol. cDNA was synthesized iScript reverse transcription kit (Bio-Rad).

Reagents for animal studies. Tamoxifen: Conditional activation of Cre recombinase was induced by oral administration of tamoxifen (Sigma). Tamoxifen (100 mg) was resuspended in $100 \mu \mathrm{l}$ of ethanol, followed by the addition of $1 \mathrm{ml}$ of sunflower oil, to a final concentration of $100 \mathrm{mg} / \mathrm{ml}$. The solution was sonicated at $4^{\circ} \mathrm{C}$ in a water bath sonicator until the tamoxifen was completely dissolved. Animals (8-9 weeks old) were administered $50 \mu \mathrm{l}(5 \mathrm{mg})$ of tamoxifen solution per day for 3 consecutive days by oral gavage.

$A A$ : AA was suspended in $120 \mathrm{~L}$ of dimethyl sulfoxide (DMSO) and then $880 \mu \mathrm{l}$ of PBS ( $\mathrm{pH} 7.4$ ), to a final concentration of $2 \mathrm{mg} / \mathrm{ml}$. The AA solution was administered to mice by intraperitoneal injection at a dosage of $5 \mathrm{mg} / \mathrm{kg}$ of body weight. Tissue was extracted for analysis 1 week after AA injection unless stated otherwise.

MK2206: MK2206 was resuspended in $1 \times$ PBS ( $\mathrm{pH} 7.4$ ), to a final concentration of $100 \mathrm{mg} / \mathrm{ml}$. The MK2206 solution was vortexed vigorously immediately prior to use. Mice were administered MK2206 by oral gavage at a dosage of $100 \mathrm{mg} / \mathrm{kg}$.

All of the necessary animal procedures were performed at the UNC mouse facility, located on the basement of the Genetic Medicine Building (GMB). The mice were housed and maintained under sterile conditions in facilities approved by the American Association for the Accreditation of Laboratory Animal Care and in accordance with current regulations and standards of the United States Department of Agriculture, United States Department of Health and Human Services, and the NIH The mice were used in accordance with Animal Care and Use Guidelines of The University of North Carolina at Chapel Hill under protocols to Drs. Pengda Liu and William Y. Kim approved by the Institutional Animal Care and Use Committee. 
Statistics. Differences between control and experimental conditions were evaluated by Student's $t$ test or one-way ANOVA. These analyses were performed using the SPSS 11.5 Statistical Software and $P \leq 0.05$ was considered statistically significant.

Reporting summary. Further information on experimental design is available in the Nature Research Reporting Summary linked to this article.

\section{Data availability}

All data supporting the findings in this study are available from the corresponding author upon reasonable request.

Received: 10 July 2018 Accepted: 27 February 2019

Published online: 03 April 2019

\section{References}

1. Li, Z. et al. The OncoPPi network of cancer-focused protein-protein interactions to inform biological insights and therapeutic strategies. Nat. Commun. 8, 14356 (2017).

2. Huttlin, E. L. et al. The BioPlex Network: a systematic exploration of the human interactome. Cell 162, 425-440 (2015).

3. Hein, M. Y. et al. A human interactome in three quantitative dimensions organized by stoichiometries and abundances. Cell 163, 712-723 (2015).

4. Rolland, T. et al. A proteome-scale map of the human interactome network. Cell 159, 1212-1226 (2014).

5. Green, D. R. A BH3 mimetic for killing cancer cells. Cell 165, 1560 (2016).

6. Manning, B. D. \& Toker, A. AKT/PKB signaling: navigating the network. Cell 169, 381-405 (2017)

7. Testa, J. R. \& Tsichlis, P. N. AKT signaling in normal and malignant cells. Oncogene 24, 7391-7393 (2005).

8. Luo, J., Manning, B. D. \& Cantley, L. C. Targeting the PI3K-Akt pathway in human cancer: rationale and promise. Cancer Cell 4, 257-262 (2003).

9. Testa, J. R. \& Bellacosa, A. AKT plays a central role in tumorigenesis. Proc. Natl. Acad. Sci. U.S.A. 98, 10983-10985 (2001).

10. Altomare, D. A. \& Testa, J. R. Perturbations of the AKT signaling pathway in human cancer. Oncogene 24, 7455-7464 (2005).

11. Sarbassov, D. D., Guertin, D. A., Ali, S. M. \& Sabatini, D. M. Phosphorylation and regulation of Akt/PKB by the rictor-mTOR complex. Science 307, 1098-1101 (2005).

12. Alessi, D. R. et al. Characterization of a 3-phosphoinositide-dependent protein kinase which phosphorylates and activates protein kinase Balpha. Curr. Biol. 7, 261-269 (1997)

13. Liu, P. et al. Cell-cycle-regulated activation of Akt kinase by phosphorylation at its carboxyl terminus. Nature 508, 541-545 (2014).

14. Guo, J. et al. pVHL suppresses kinase activity of Akt in a prolinehydroxylation-dependent manner. Science 353, 929-932 (2016).

15. Sundaresan, N. R. et al. The deacetylase SIRT1 promotes membrane localization and activation of Akt and PDK1 during tumorigenesis and cardiac hypertrophy. Sci. Signal. 4, ra46 (2011).

16. Yang, W. L. et al. The E3 ligase TRAF6 regulates Akt ubiquitination and activation. Science 325, 1134-1138 (2009).

17. Chan, C. H. et al. The Skp2-SCF E3 ligase regulates Akt ubiquitination, glycolysis, herceptin sensitivity, and tumorigenesis. Cell 149, 1098-1111 (2012).

18. Xie, J., Wang, X. \& Proud, C. G. mTOR inhibitors in cancer therapy. F1000Res. 5, 2078 (2016).

19. Du, K. \& Tsichlis, P. N. Regulation of the Akt kinase by interacting proteins. Oncogene 24, 7401-7409 (2005).

20. Jin, L., Wang, W. \& Fang, G. Targeting protein-protein interaction by small molecules. Annu. Rev. Pharmacol. Toxicol. 54, 435-456 (2014).

21. Lloyd, A. C. The regulation of cell size. Cell 154, 1194-1205 (2013).

22. Yu, F. X., Zhao, B. \& Guan, K. L. Hippo pathway in organ size control, tissue homeostasis, and cancer. Cell 163, 811-828 (2015).

23. Salah, Z. \& Aqeilan, R. I. WW domain interactions regulate the Hippo tumor suppressor pathway. Cell Death Dis. 2, e172 (2011).

24. Ingham, R. J. et al. WW domains provide a platform for the assembly of multiprotein networks. Mol. Cell. Biol. 25, 7092-7106 (2005).

25. Leung, J. Y. et al. Sav1 loss induces senescence and Stat3 activation coinciding with tubulointerstitial fibrosis. Mol. Cell. Biol. 37, e00565-16 (2017).

26. Matsuura, K. et al. Downregulation of SAV1 plays a role in pathogenesis of high-grade clear cell renal cell carcinoma. BMC Cancer 11, 523 (2011).

27. Monzon, F. A. et al. Chromosome 14q loss defines a molecular subtype of clear-cell renal cell carcinoma associated with poor prognosis. Mod. Pathol. 24, 1470-1479 (2011).
28. Tapon, N. et al. Salvador promotes both cell cycle exit and apoptosis in Drosophila and is mutated in human cancer cell lines. Cell 110, 467-478 (2002).

29. Tumaneng, K. et al. YAP mediates crosstalk between the Hippo and PI(3)KTOR pathways by suppressing PTEN via miR-29. Nat. Cell Biol. 14, 1322-1329 (2012).

30. Meng, Z. et al. MAP4K family kinases act in parallel to MST1/2 to activate LATS1/2 in the Hippo pathway. Nat. Commun. 6, 8357 (2015).

31. Yu, F. X. et al. Regulation of the Hippo-YAP pathway by G-protein-coupled receptor signaling. Cell 150, 780-791 (2012).

32. Schlegel, J. et al. MERTK receptor tyrosine kinase is a therapeutic target in melanoma. J. Clin. Invest. 123, 2257-2267 (2013)

33. Graham, D. K., DeRyckere, D., Davies, K. D. \& Earp, H. S. The TAM family: phosphatidylserine sensing receptor tyrosine kinases gone awry in cancer. Nat. Rev. Cancer 14, 769-785 (2014).

34. Rankin, E. B. et al. Direct regulation of GAS6/AXL signaling by HIF promotes renal metastasis through SRC and MET. Proc. Natl Acad. Sci. USA 111, 13373-13378 (2014)

35. Nguyen, K. Q. et al. Overexpression of MERTK receptor tyrosine kinase in epithelial cancer cells drives efferocytosis in a gain-of-function capacity. J. Biol. Chem. 289, 25737-25749 (2014).

36. Liu, P. et al. PtdIns $(3,4,5)$ P3-dependent activation of the mTORC2 kinase complex. Cancer Discov. 5, 1194-1209 (2015)

37. Li, L. \& Hanahan, D. Hijacking the neuronal NMDAR signaling circuit to promote tumor growth and invasion. Cell 153, 86-100 (2013).

38. Yap, T. A. et al. First-in-man clinical trial of the oral pan-AKT inhibitor MK-2206 in patients with advanced solid tumors. J. Clin. Oncol. 29, 4688-4695 (2011).

39. Shao, W. H. et al. A protective role of Mer receptor tyrosine kinase in nephrotoxic serum-induced nephritis. Clin. Immunol. 136, 236-244 (2010).

40. Birge, R. B. et al. Phosphatidylserine is a global immunosuppressive signal in efferocytosis, infectious disease, and cancer. Cell Death Differ. 23, 962-978 (2016).

41. Bester, A. C. et al. An integrated genome-wide CRISPRa approach to functionalize lncRNAs in drug resistance. Cell 173, 649-664 (2018).

42. Zhang, J. et al. VHL substrate transcription factor ZHX2 as an oncogenic driver in clear cell renal cell carcinoma. Science 361, 290-295 (2018).

43. Liu, P. et al. Akt-mediated phosphorylation of XLF impairs non-homologous end-joining DNA repair. Mol. Cell 57, 648-661 (2015).

44. Kozakov, D. et al. The ClusPro web server for protein-protein docking. Nat. Protoc. 12, 255-278 (2017).

45. Kozakov, D. et al. How good is automated protein docking? Proteins 81, 2159-2166 (2013).

46. Kozakov, D., Brenke, R., Comeau, S. R. \& Vajda, S. PIPER: an FFT-based protein docking program with pairwise potentials. Proteins 65, 392-406 (2006).

47. Comeau, S. R., Gatchell, D. W., Vajda, S. \& Camacho, C. J. ClusPro: an automated docking and discrimination method for the prediction of protein complexes. Bioinformatics 20, 45-50 (2004).

48. Comeau, S. R., Gatchell, D. W., Vajda, S. \& Camacho, C. J. ClusPro: a fully automated algorithm for protein-protein docking. Nucleic Acids Res. 32 W96-W99 (2004).

49. Thomas, C. C., Deak, M., Alessi, D. R. \& van Aalten, D. M. High-resolution structure of the pleckstrin homology domain of protein kinase b/akt bound to phosphatidylinositol (3,4,5)-trisphosphate. Curr. Biol. 12, 1256-1262 (2002).

50. Shirvanyants, D., Ding, F., Tsao, D., Ramachandran, S. \& Dokholyan, N. V. Discrete molecular dynamics: an efficient and versatile simulation method for fine protein characterization. J. Phys. Chem. B 116, 8375-8382 (2012).

51. Ding, F., Tsao, D., Nie, H. \& Dokholyan, N. V. Ab initio folding of proteins with all-atom discrete molecular dynamics. Structure 16, 1010-1018 (2008).

52. Dokholyan, N. V., Buldyrev, S. V., Stanley, H. E. \& Shakhnovich, E. I. Discrete molecular dynamics studies of the folding of a protein-like model. Fold. Des. 3 577-587 (1998)

53. Yin, S., Ding, F. \& Dokholyan, N. V. Eris: an automated estimator of protein stability. Nat. Methods 4, 466-467 (2007).

54. Yin, S., Ding, F. \& Dokholyan, N. V. Modeling backbone flexibility improves protein stability estimation. Structure 15, 1567-1576 (2007).

\section{Acknowledgements}

The authors thank Liu, Earp, and Kim lab members for critical reading of the manuscript and helpful discussions. The authors also thank Dr. Qing Zhang (UNC-Chapel Hill) and Zhang lab members for sharing the reagents and suggestions. This work was supported by the NIH grants (P.L. R00CA181342, H.S.E. CA016086, N.V.D. R01GM114015 and R01GM123247), the UNC IBM Junior Faculty Development Award (P.L.), the V Scholar Research Grant (P.L.), and UNC University Cancer Research Fund (P.L.).

\section{Author contributions}

Conceptualization: W.Y.K., H.S.E., and P.L. Methodology: N.V.D. and C.M.P. Investigation: Y.J., Y.Z., J.Y.L., C.F., K.I.P., S.S., J.Q., X.W., A.H.H., E.U., and Y.X. Writing- 
original draft: Y.J., Y.Z., and P.L. Writing-review \& editing: I.D., W.Y.K., K.I.P., and H. S.E. Funding acquisition: N.V.D., G.W., C.M.P., W.Y.K., H.S.E., and P.L. Resources: X. W., N.V.D., G.W., and C.M.P. Supervision: W.Y.K., H.S.E., and P.L.

\section{Additional information}

Supplementary Information accompanies this paper at https://doi.org/10.1038/s41467019-09233-7.

Competing interests: H.S.E. is a founder of Meryx (a UNC startup) that is developing small molecule inhibitors for MERTK. H.S.E. and X.W. own stock in Meryx. The other authors declare no competing interests.

Reprints and permission information is available online at http://npg.nature.com/ reprintsandpermissions/

Journal peer review information: Nature Communications thanks James Hsieh and the other anonymous reviewer(s) for their contribution to the peer review of this work. Peer reviewer reports are available.
Publisher's note: Springer Nature remains neutral with regard to jurisdictional claims in published maps and institutional affiliations.

(c) (i) Open Access This article is licensed under a Creative Commons Attribution 4.0 International License, which permits use, sharing, adaptation, distribution and reproduction in any medium or format, as long as you give appropriate credit to the original author(s) and the source, provide a link to the Creative Commons license, and indicate if changes were made. The images or other third party material in this article are included in the article's Creative Commons license, unless indicated otherwise in a credit line to the material. If material is not included in the article's Creative Commons license and your intended use is not permitted by statutory regulation or exceeds the permitted use, you will need to obtain permission directly from the copyright holder. To view a copy of this license, visit http://creativecommons.org/ licenses/by/4.0/.

(c) The Author(s) 2019 